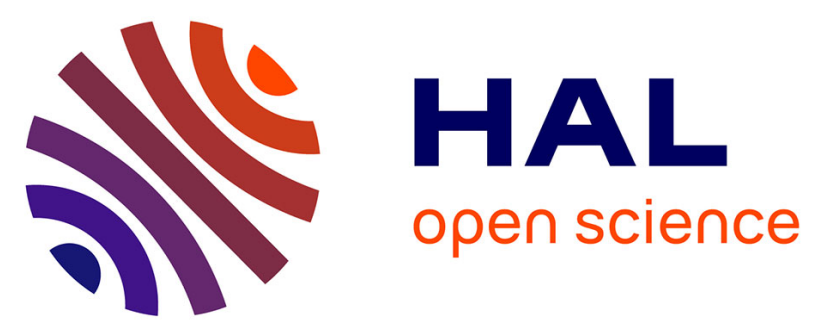

\title{
Application of differential effective medium, magnetic pore fabric analysis, and X-ray microtomography to calculate elastic properties of porous and anisotropic rock aggregates
}

Bjarne S. G. Almqvist, David Mainprice, Claudio Madonna, Luigi Burlini, Ann M. Hirt

\section{To cite this version:}

Bjarne S. G. Almqvist, David Mainprice, Claudio Madonna, Luigi Burlini, Ann M. Hirt. Application of differential effective medium, magnetic pore fabric analysis, and X-ray microtomography to calculate elastic properties of porous and anisotropic rock aggregates. Journal of Geophysical Research, 2011, 116, pp.B01204. 10.1029/2010JB007750 . hal-00617717

\section{HAL Id: hal-00617717 https://hal.science/hal-00617717}

Submitted on 30 Apr 2021

HAL is a multi-disciplinary open access archive for the deposit and dissemination of scientific research documents, whether they are published or not. The documents may come from teaching and research institutions in France or abroad, or from public or private research centers.
L'archive ouverte pluridisciplinaire HAL, est destinée au dépôt et à la diffusion de documents scientifiques de niveau recherche, publiés ou non, émanant des établissements d'enseignement et de recherche français ou étrangers, des laboratoires publics ou privés. 


\title{
Application of differential effective medium, magnetic pore fabric analysis, and $X$-ray microtomography to calculate elastic properties of porous and anisotropic rock aggregates
}

\author{
Bjarne S. G. Almqvist, ${ }^{1}$ David Mainprice, ${ }^{2}$ Claudio Madonna, ${ }^{3}$ Luigi Burlini, ${ }^{3}$ \\ and Ann M. Hirt ${ }^{1}$ \\ Received 3 June 2010; revised 21 September 2010; accepted 20 October 2010; published 14 January 2011.
}

[1] A differential effective medium (DEM) model is used to predict elastic properties for a set of porous and anisotropic aggregates, comprised of mixtures of calcite and muscovite. The DEM takes into consideration an anisotropic background medium with triclinic or higher symmetry, in which inclusions of idealized ellipsoidal shape are added incrementally. In general, the calculated elastic properties of a solid that contains inclusions representing "dry" pores/cracks are strongly dependent on the orientation and aspect ratio of the inclusions. Aspect ratios of inclusions in the synthetic aggregates, which consist of air-filled pores, are estimated from anisotropy of magnetic susceptibility (AMS) of samples whose pore space has been impregnated with a colloidal ferrofluid. The AMS derived pore shape geometry is used as an input value for inclusions in the DEM. Modeling results are compared with laboratory determined elastic properties, measured with ultrasonic waves. Calculated shear wave velocities agree in general well with laboratory measured $S$ wave velocities, whereas calculated $P$ wave velocities are typically $0.5-1.1 \mathrm{~km} / \mathrm{s}$ higher than measured values. Differences between calculated and measured $P$ wave velocities are attributed mainly to incomplete and biased ferrofluid saturation of pores. Spherical pores are preferably filled during imbibition, in comparison to thin cracks, which leads to overprediction of the calculated $P$ wave velocities. The amount of ferrofluid that fills the pore space is dependent on the ratio of calcite to muscovite and the load used for compaction during sample synthesis. The permeability decreases with increasing muscovite content and increasing compaction load. Incomplete saturation of samples with high-muscovite content is confirmed by X-ray microtomography density contrast imaging of dry and ferrofluid saturated specimens.

Citation: Almqvist, B. S. G., D. Mainprice, C. Madonna, L. Burlini, and A. M. Hirt (2011), Application of differential effective medium, magnetic pore fabric analysis, and X-ray microtomography to calculate elastic properties of porous and anisotropic rock aggregates, J. Geophys. Res., 116, B01204, doi:10.1029/2010JB007750.

\section{Introduction}

[2] Effective medium theory is commonly used to predict seismic velocities in rocks from various geological settings, taking into account the crystallographic and shape-preferred orientation of individual components in the composite material, their relative amounts, and their individual elastic properties [Mavko et al., 2009]. Several modeling schemes are presently available for this purpose, and a large body of literature in the physical and engineering sciences has accumulated. The most general case for a geological material requires information on the volume fractions of each com-

\footnotetext{
${ }^{1}$ Institute of Geophysics, ETH Zurich, Zurich, Switzerland.

${ }^{2}$ Géosciences Montpellier, Université Montpellier 2, Montpellier, France.

${ }^{3}$ Geological Institute, ETH Zurich, Zurich, Switzerland.
}

Copyright 2011 by the American Geophysical Union. 0148-0227/11/2010JB007750 ponent (e.g., mineral phases) and their individual elastic properties (bulk and shear moduli). With this information it is possible to specify upper and lower bounds of the elastic moduli (i.e., Voigt and Reuss bounds, Hashin-Shtrikman bounds). However, further knowledge regarding the orientation distribution of minerals grains and inclusions can be used to provide more rigorous bounds for the calculated velocities, applicable to anisotropic elastic materials. Rocks in many geological settings are more complicated than current models can account for despite considering these additional parameters. Nevertheless, seismic velocity prediction with effective medium theory is an important tool in rock physics. Empirical, heuristic, and purely theoretical models that predict seismic velocities are frequently used in the petroleum industry [cf. Avseth et al., 2005], and in geodynamical models of the low-velocity zone at mid-ocean ridges [Walsh, 1969; Anderson et al., 1974; Mainprice, 1997], seismic anisotropy in subducting plates [Faccenda et al., 2008; Healy et al., 2009], dilatation phenomena occurring 
during earthquakes [e.g., Le Ravalec et al., 1996], and seismic properties of other deep Earth settings [e.g., Wenk et al., 1988; Kendall and Silver, 1996; Berryman, 2000; Mainprice, 2007]. In many cases effective medium theory is useful to help predict seismic wave propagation in rocks ranging from the Earth's crust to the core, for which laboratory derived elastic properties are not available.

[3] Various methods have been developed and employed to calculate elastic properties in porous media [see Mavko et al., 2009]. Most models consider an isotropic background matrix, even though this is an exceptional case for mineral aggregates in most crystalline rocks. The simplest and most rigorous predictions are Voigt and Reuss bounds, which provide upper and lower constraints on the elastic constants [Voigt, 1928; Reuss, 1929; Hill, 1952], but these consider only the volume fractions of each elastic component, which may have a crystallographic preferred orientation (CPO), but no shape-preferred orientation (SPO). When the difference in elastic properties between the two components (e.g., solid and gas [Mavko et al., 2009]) is large, or the elastic anisotropy of components is strong (e.g., mica [Mainprice and Humbert, 1994]), widely separated upper and lower bounds are found.

[4] A more precise prediction of elastic properties can be made using a self-consistent (SC) effective medium model. Such a model considers the mixture of two or more components in the same manner as discussed above. Inclusions of these components are inserted to an unknown background medium, which is assigned an initial value (e.g., the Voigt average), and the SC solution is found by iteration. O'Connell and Budiansky [1974] popularized the SC approximation, by which the elastic moduli of dry or wet inclusions of specified shapes could be precisely calculated. Henyey and Pomphrey [1982] have, however, criticized the SC method by showing that the scheme developed by O'Connell and Budiansky does not properly account for interaction among cracks. This has the effect of overestimating the properties of the inclusion, particularly for high concentrations. They suggested instead using a modified self-consistent approximation whereby the elastic moduli are calculated using differential equations, appropriately termed the differential self-consistent approximation or differential effective medium (DEM). This method was initially used by Bruner [1976] for prediction of seismic velocities in geological materials. McLaughlin [1977] showed that DEM lies between the Hashin-Shtrikman bounds for spherical inclusions.

[5] The DEM is based on two components; the background and the inclusion. Inclusions, of specific geometry, are added incrementally in small amounts and the elastic properties of the effective medium are continuously updated before another increment of the inclusion is added. In the current work we employ the DEM to compute elastic properties for calcite and muscovite mixtures. The current work differs from previous effective medium studies in the following ways: (1) Most previous work considers the background medium to be isotropic, whereas in this study the DEM is computed for an anisotropic background medium [Mainprice, 1997]; and (2) the effective medium for large volume porosities are computed, which is suitable for highly porous sedimentary rocks and unconsolidated sediments. Furthermore we compare the predicted seismic velocities with laboratory seismic velocity measurements on synthetic calcite-muscovite aggregates to test the DEM [Almqvist et al., 2010]. These aggregates have been synthesized by compaction [Schmidt et al., 2008], resulting in transverse isotropic sample symmetry, although a material with lower symmetry, and hence a larger number of independent elastic constants, can also be considered. Samples with $15 \mathrm{~mm}$ diameter and $13 \mathrm{~mm}$ length were drilled from the center of $\sim 5 \mathrm{~cm}$ diameter canisters, in order to minimize sample heterogeneity and edge effects that may occur during synthesis. Seismic velocity measurements were made on oven-dried specimens [Almqvist et al., 2010]. The physical characteristics of these synthetic rocks are well defined and single-crystal elastic properties, modal fractions for calcite and muscovite, and their respective orientation distribution functions (ODF) are well-constrained [Schmidt et al., 2009; Almqvist et al., 2010]. The porosity has been measured, and the pore shape geometry was mapped by impregnating the samples with ferrofluids measuring their anisotropy of magnetic susceptibility [e.g., Pfleiderer and Halls, 1990, 1993, 1994; Hrouda et al., 2000; Benson et al., 2003; Louis et al., 2005; Jones et al., 2006].

\section{Modeling Schemes}

[6] Calculation of the elastic properties is performed in two steps. First, the elastic constants for the solid matrix are computed and second, the "dry" inclusions are added to the solid matrix. A third step (not treated in this paper) is fluid substitution, which can be made appropriately using Gassmann's equation (Figure 1) [Gassmann, 1951; Biot, 1956].

\subsection{The Solid Matrix}

[7] The solid matrix serves as the starting or background material, prior to adding pore or cracklike inclusions. It is anisotropic and described by a fourth rank elastic tensor, enabling the consideration of low-symmetry cases. Elastic moduli for the matrix are calculated based on single-crystal elastic tensors of the minerals present in the sample, as well as their crystallographic preferred orientation [Mainprice and Nicolas, 1989; Mainprice, 1990; Mainprice and Humbert, 1994]. In addition, the modal abundance for each phase in the rock has to be incorporated into the calculation.

[8] Appropriate single-crystal elastic moduli have been taken from the literature; for calcite we use elastic constants determined by Dandekar [1968] and Chen et al. [2001], and for muscovite the elastic constants provided by Vaughan and Guggenheim [1986]. Both calcite and muscovite are highly anisotropic with $V_{P}$ anisotropy above $30 \%$ and shear wave splitting greater than $50 \%$ for single crystals (Figure 2 ). The modeled acoustic velocities for the solid matrix have been discussed in detail by Almqvist et al. [2010].

\subsection{The Differential Effective Medium}

[9] As discussed above, the DEM is based on adding inclusions in small, incremental steps, while continuously updating the elastic constants for the effective medium between each increment. The DEM is restricted to the mixing of two media, in contrast to the iterative self-consistent method, which can treat the mixing of more than two media. However, two materials are often enough to model a geological system, and if more than two materials need to be considered it is possible to do the calculation by mixing 
1. Computation of elastic constants for solid matrix
2. Adding inclusions with the DEM
3. Partially or fully saturate the medium using Gassmann's equation

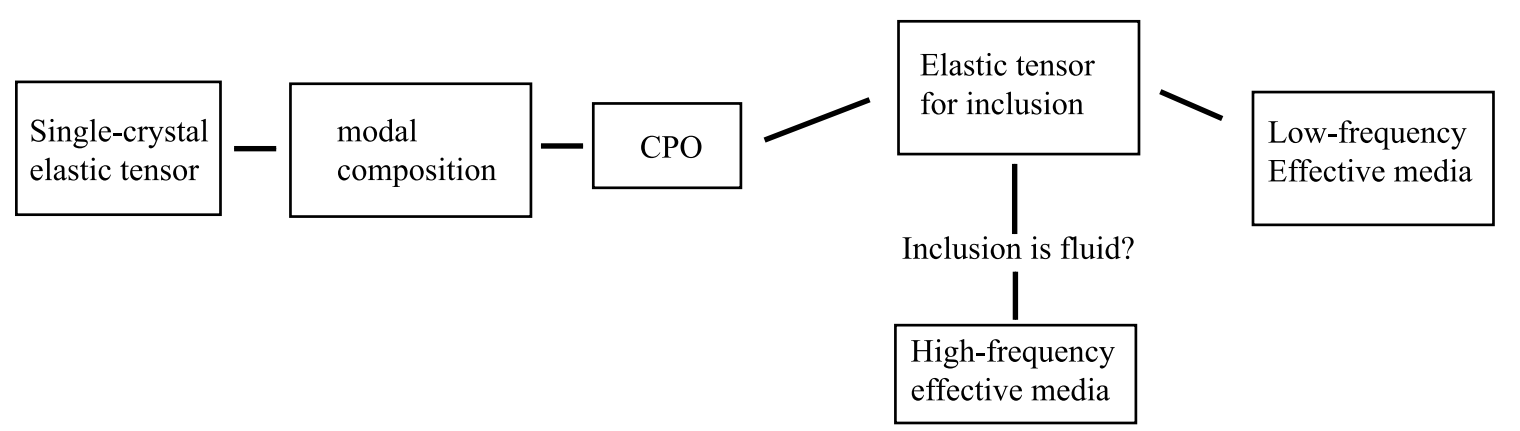

Figure 1. Flowchart of the modeling procedure.

(a) Vp Muscovite single-crystal

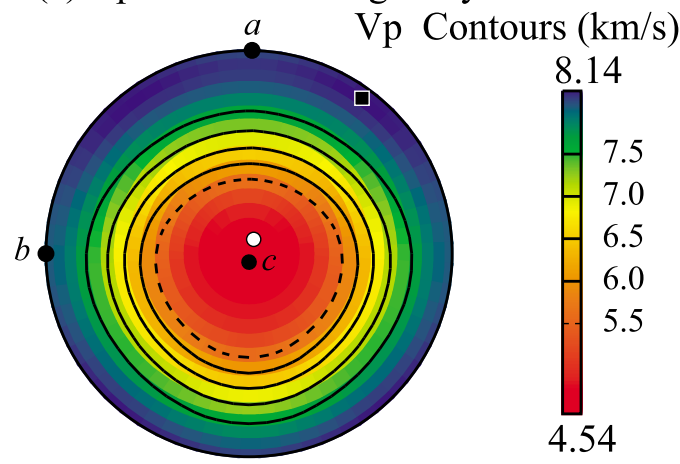

$\bigcirc$ Min.Velocity $=4.54$

- Max.Velocity $=8.14$

Anisotropy $=56.7 \%$

(b) dVs Muscovite single-crystal

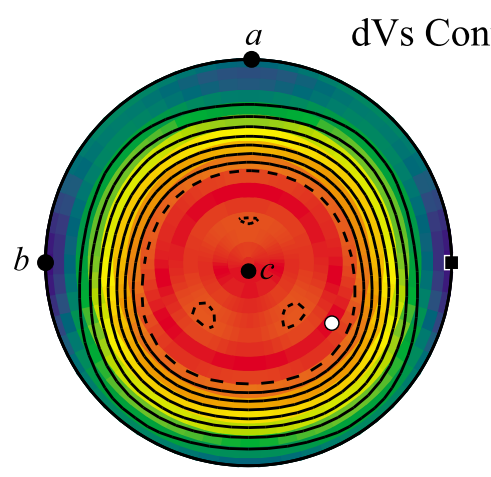

$\mathrm{OMin} . \mathrm{dVs}=.01$

Max.dVs $=2.68$ (c) Vp Calcite single-crystal

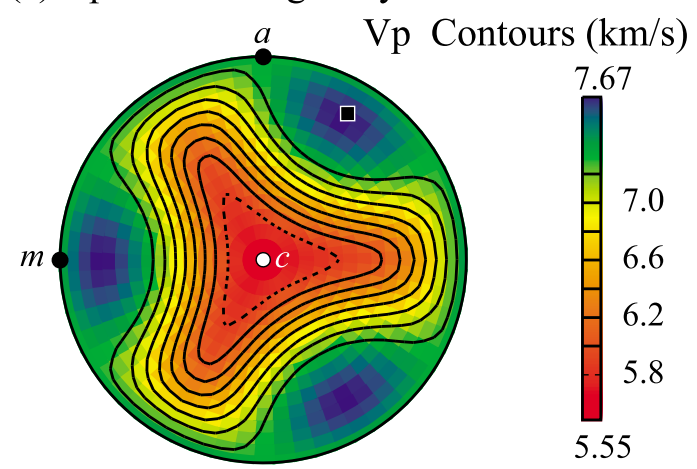

OMin.Velocity $=5.55$

Max.Velocity $=7.67$

Anisotropy $=32.1 \%$

(d) dVs Calcite single-crystal

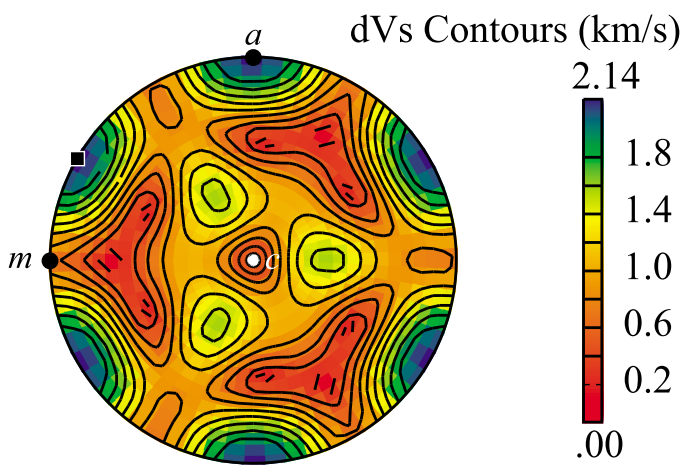

$\mathrm{OMin} \cdot \mathrm{dVs}=.00$

-Max.dVs $=2.14$

Figure 2. Equal-area projections for compressional wave velocity of single crystals of (a) muscovite and (c) calcite and for maximum shear wave splitting $\left(\mathrm{dV}_{\mathrm{S}}\right)$ in (b) muscovite and (d) calcite. Note the orientation of the crystallographic $a, b$, and $c$ axes; $m$ is the pole to the trigonal prism. The angle between the crystallographic $a$ and $c$ axes in muscovite is $\sim 95.5^{\circ}$, arising from its monoclinic symmetry. 
two media, and applying the resulting media in subsequent DEM calculations. The DEM is computed using the formulation derived by McLaughlin [1977] for anisotropic elastic media where

$$
\frac{d C^{\mathrm{DEM}}}{d V}=\frac{1}{(1-V)}\left(C_{i}-C^{\mathrm{DEM}}\right) A_{i},
$$

where $C^{\text {DEM }}$ is the elastic tensor of the effective media, $V$ is the volume fraction of the inclusion, $C_{i}$ is the elastic tensor of the inclusion, and $A_{i}$ is a term that relates the strain inside the inclusion with the strain of the background matrix [Willis, 1977], which is defined as

$$
A_{i}=\left[I+G\left(C_{i}-C^{\mathrm{DEM}}\right)\right]^{-1} .
$$

In equation (2) $I$ is the symmetric fourth rank unit tensor, $G$ is the symmetric tensor Green's function [Barnett, 1972; Mura, 1987], $C_{i}$ is the elastic moduli of the inclusion, and $C^{\text {DEM }}$ is the elastic moduli of the DEM, which is updated between each inclusion step. The integration of the tensor Green's function is done numerically, by taking the Fourier transform of $G$ with the symmetry of a triaxial ellipsoid [Barnett, 1972]. Inclusions are idealized ellipsoids with an arbitrary aspect ratio $(\mathrm{a} \geq \mathrm{b} \geq \mathrm{c})$, where strain and stress are uniform at all points inside the inclusion [Eshelby, 1957]. The DEM requires the solution to be computed numerically, because of the arbitrary symmetry conditions imposed on the background matrix. As a result, when aspect ratios are large, i.e., $\mathrm{a}=\mathrm{b} \gg \mathrm{c}$, the computation becomes more computation intensive. In return, any background symmetry can be considered, as well as any orientation and aspect ratio of the inclusions, providing a flexible combination to compute the effective medium. To illustrate the use of the DEM we have chosen two synthetic samples with well-defined mineral composition, texture (CPO), and porosity. The first material is a nearly isotropic calcite aggregate that was compacted with a uniaxial stress of $20 \mathrm{MPa}$, and the second material is an aggregate of muscovite, which has a high intrinsic anisotropy [Schmidt et al., 2008, 2009; Almqvist et al., 2010].

[10] Le Ravalec and Guéguen [1996] used the DEM to compute elastic constants for an isotropic solid matrix with inclusions of arbitrary ellipsoids. They were limited to aspect ratios, $\alpha$, of unity (spherical pores) or $\geq 10$ (aspect ratios between 1 and 10 were thus not considered), where $\alpha$ is defined as $\alpha=\mathrm{a} / \mathrm{c}$, and $\mathrm{b}=\mathrm{a}$, for a rotational oblate ellipsoid. The DEM used in this study is able to consider any aspect ratio of the inclusion including spherical pores. At low volume fractions of pores or cracks (1-5\%), which are likely to be more realistic for most crystalline rocks, the velocity changes are more pronounced for high aspect ratios in $V_{P}$ propagation direction normal to the crack symmetry plane $Z$, whereas $d V_{S}$ changes are more modest [e.g., Benson et al., 2006a, 2006b]. In contrast for porosities typical of reservoir rocks $(20-40 \%) V_{P}$ and $V_{S}$ changes are already significant for aspect ratios 1 to 5 . For high porosities characteristic of poorly compacted sediments (40-80\%) the effect of the low aspect ratio is of particular importance for $d V_{S}$, which may be significant for carbonate reservoir rocks, such as chalk and mudrocks [Urmos and Wilkens, 1993; Røgen et al., 2001; Mallon et al., 2005]. Figures 3a and 3c display a scenario where we consider an aggregate of crystals, which all have the same orientation, effectively producing the same result as the elastic tensor of a single crystal. This can be considered an end-member upper bound for anisotropic medium. A perfect arrangement of crystals is very unlikely in a natural setting, although this scenario displays the effect of anisotropy of the solid matrix, and strong CPOs can certainly result from natural deformation processes. The strong influence of CPO is illustrated in Figures $3 \mathrm{e}$ and $3 \mathrm{f}$, where compacted muscovite and calcite aggregates show different velocities parallel and normal to the axis of compaction, as well as from their intrinsic elastic single-crystal anisotropies. This is mainly seen in the anisotropy of calcite, for which the synthetic aggregates display nearly no anisotropy due to the matrix compared to nearly $30 \%$ anisotropy for the single crystal (i.e., Figures $3 \mathrm{~b}$ and $3 \mathrm{f}$ ). Shear wave splitting ( $d V_{S}$; Figures $3 \mathrm{c}$ and $\left.3 \mathrm{~d}\right)$ is also affected by the variation in crack aspect ratio, with the added effect of anisotropy of the solid matrix. Notably, the evolution of $d V_{S}$ with increasing porosity is not simple in either material, but the high intrinsic anisotropy of the muscovite leads to a very large shear wave splitting in the muscovite sample. The impact of CPO and cracklike inclusions is shown also in synthetic aggregates of muscovite and calcite, for which the anisotropy of the solid matrix is known (Figures $3 \mathrm{e}-3 \mathrm{~h}$ ). In these two samples it is notable that when aspect ratios of the included pores are small the anisotropy tends to decrease with increasing porosity in the case of muscovite (Figures 3e and $3 \mathrm{~g}$ ). For the calcite sample (Figures $3 \mathrm{f}$ and $3 \mathrm{~h}$ ), which has a very weak matrix anisotropy due to its $\mathrm{CPO}$, pores that are spherical do not noticeably affect the seismic anisotropy, whereas pores that have slightly ellipsoidal aspect ratio (e.g., $\alpha=2$ ) increase the seismic anisotropy as the porosity increases. The modeling results for the nearly isotropic calcite indicate that minor differences between the principal axes of the inclusion will have a considerable impact on the seismic anisotropy.

[11] In Figure 4, $P$ wave velocity and shear wave splitting are shown as a function of crack geometry, for cracks that have their long axes oriented normal to the symmetry axis ( $Z$ axis) of the muscovite and calcite samples; the porosity is kept constant at $10 \%$. $V_{P}$ varies most with respect to the aspect ratio when the waves propagate along the axis of symmetry, whereas the effect due to cracks is much less

Figure 3. Compressional waves that propagate parallel $\left(V_{P} X\right)$ (solid curve) and normal $\left(V_{P} Z\right)$ (dashed curve) to the sample symmetry axis in single crystals of (a) muscovite and (b) calcite that contains porous inclusions of varying aspect ratio $(\alpha)$. Muscovite has its basal plane parallel to the $X-Y$ plane, whereas for calcite the crystallographic $a$ axes are parallel to the $X-Y$ plane. The long axes of the pores are oriented normal to the sample symmetry axis ( $Z$ axis). (c and d) The maximum shear wave splitting $\left(d V_{S}\right)$ is shown for single crystals of muscovite and calcite, with dry inclusions of varying aspect ratios. Similarly, $P$ wave velocity and $d V_{S}$ are displayed for synthetic, compacted samples of (e, g) muscovite and (f, h) calcite with waves propagating parallel to the sample symmetry axis. 


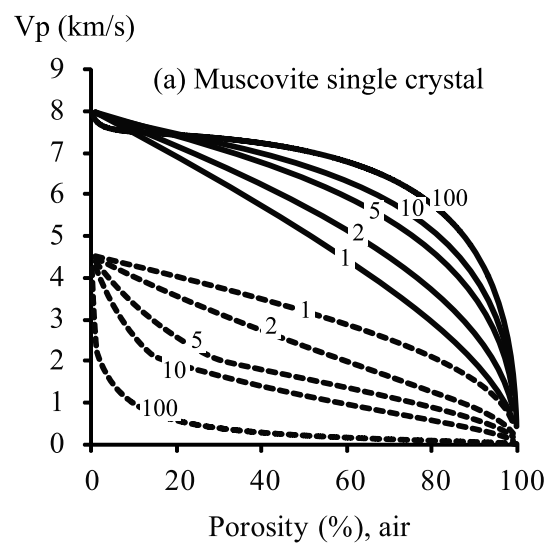

$\operatorname{Max} \mathrm{dVs}(\mathrm{km} / \mathrm{s})$

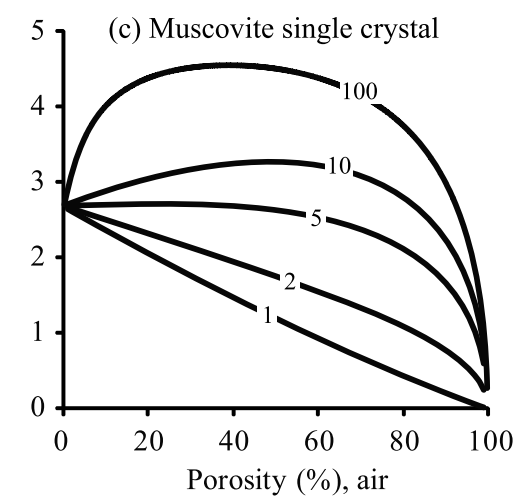

$\mathrm{Vp}(\mathrm{km} / \mathrm{s})$

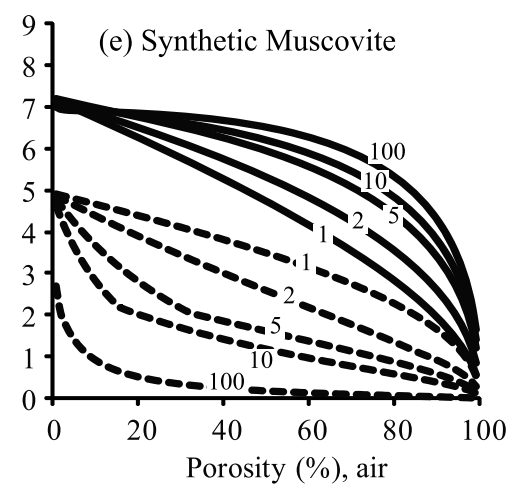

Max dVs $(\mathrm{km} / \mathrm{s})$

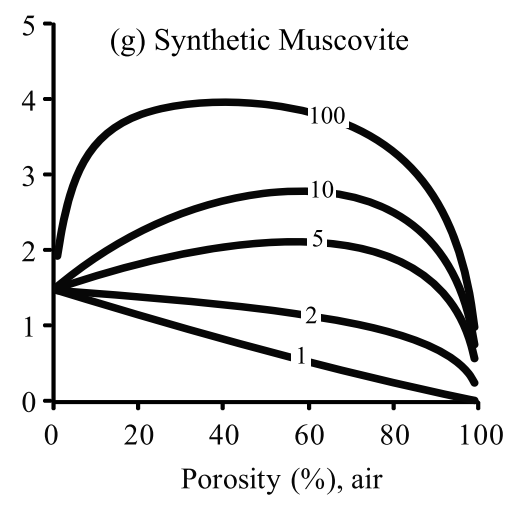

$\mathrm{Vp}(\mathrm{km} / \mathrm{s})$

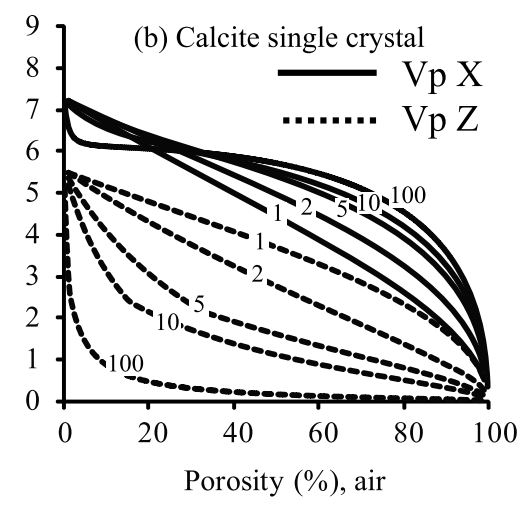

$\operatorname{Max} \mathrm{dVs}(\mathrm{km} / \mathrm{s})$

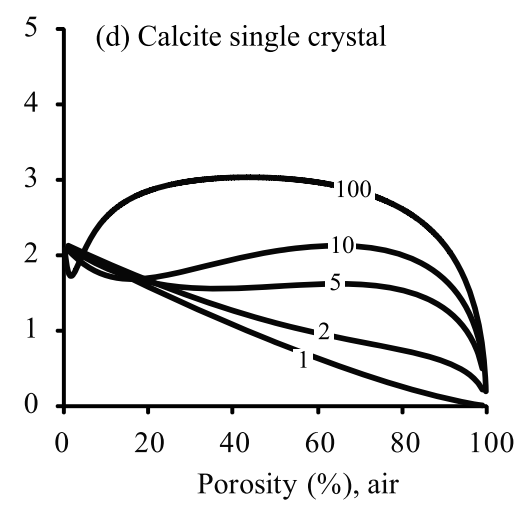

$\mathrm{Vp}(\mathrm{km} / \mathrm{s})$

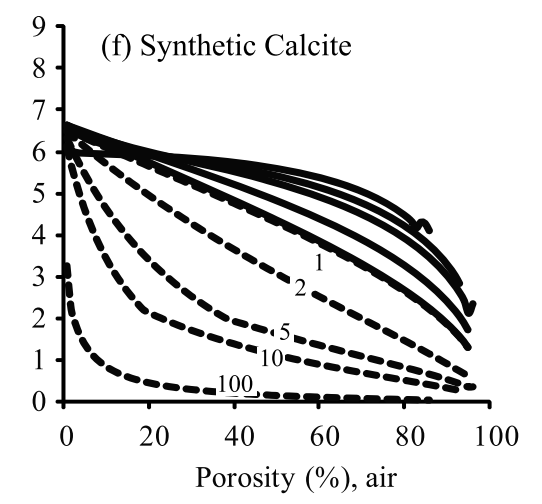

$\operatorname{Max} \mathrm{dVs}(\mathrm{km} / \mathrm{s})$

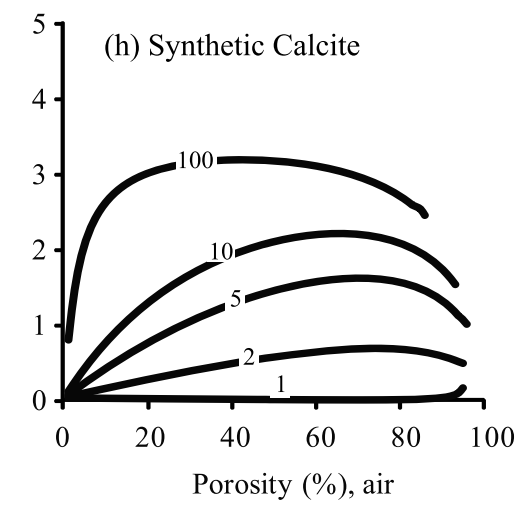

Figure 3
Vp Z (axis of symmetry)

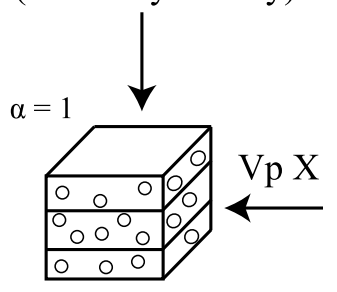

$\alpha=10$
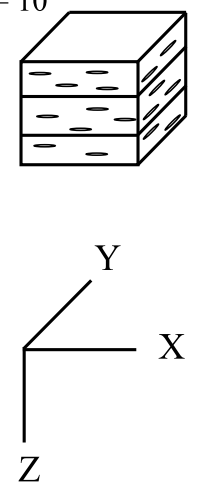

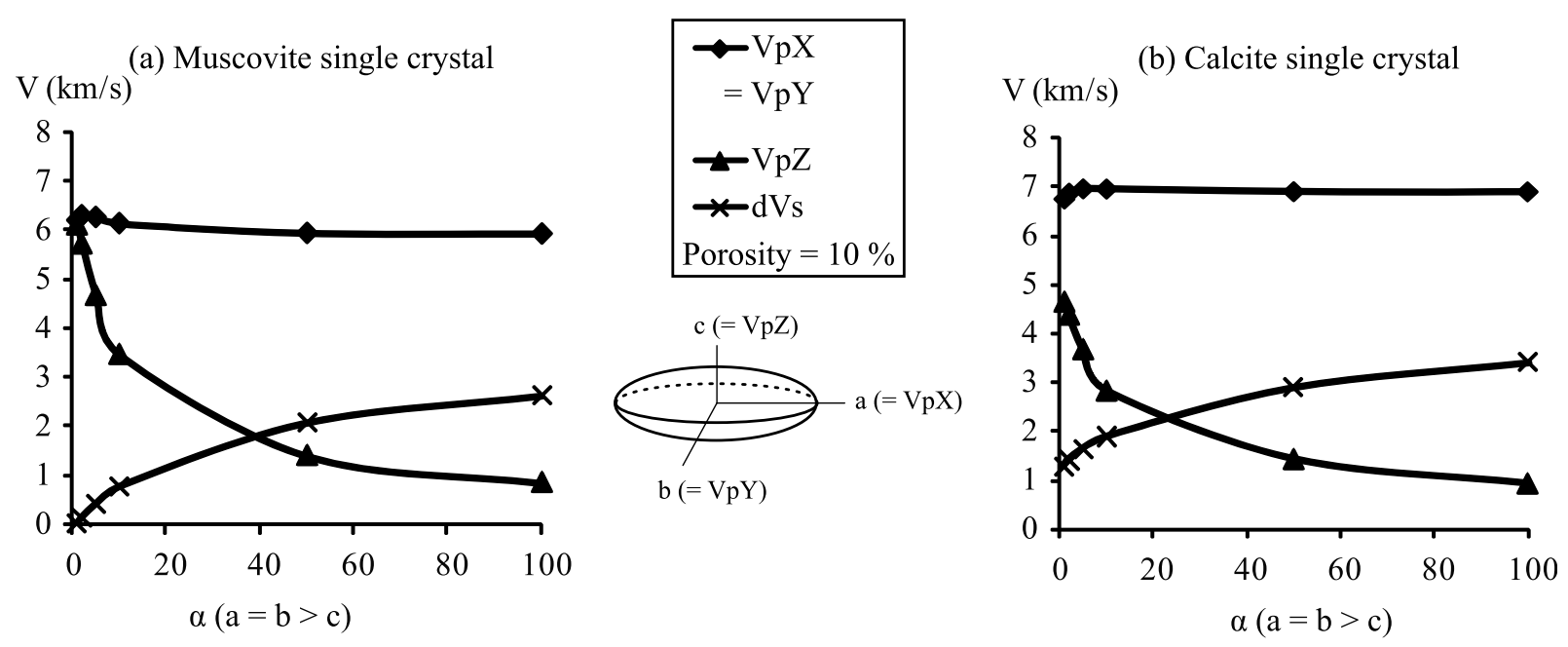

Figure 4. Compressional wave velocity and maximum shear wave splitting $\left(d V_{S}\right)$ as a function of varying pore aspect ratio, $\alpha$, for single crystals of (a) muscovite and (b) calcite at constant porosity $(\varphi=10 \%)$. $\mathrm{X}, \mathrm{Y}$, and $\mathrm{Z}$ refer to orthogonal sample $X, Y$, and $Z$ axes, where $\mathrm{X}$ and $\mathrm{Y}$ are oriented in the plane normal to the symmetry axis of the sample, and $\mathrm{Z}$ is oriented parallel to the axis of symmetry.

evident in the plane normal to the symmetry axis. The compressional waves that propagate in this plane do not effectively "see" the cracks. The shear wave splitting in the $X-Y$ plane, however, increases as the aspect ratio of the crack becomes higher (i.e., cracks become flatter). Elastic wave anisotropy is reinforced at low to medium porosities when bedding/foliation parallel cracks form.

[12] Another considered case is where dilatant cracks form perpendicular to the bedding (i.e., with their long axes parallel to the compaction axis; Figure 5). There the cracks have their short axes parallel to the symmetry axis of the transverse isotropic solid matrix ( $X$ axis), whereas the long axes of the cracks are oriented in the $Y-Z$ plane normal to the short axis of the crack (see inset of Figure 5); $\alpha=10$. The combination of an anisotropic solid matrix and planar cracks with orientations perpendicular to the transverse isotropic symmetry of the matrix give rise to a complicated behavior of the compressional seismic velocities along the three principal axes $(X, Y$, and $Z)$, and of the maximum observed shear wave splitting $\left(d V_{S}\right)$ in the effective medium. The $V_{P}$ in the $X$ direction shows very strong influence of the cracks on propagation in the direction normal to the crack symmetry plane, which mimics the behavior previously documented for the $Z$ direction in Figure 3. The decrease of $d V_{S}$ for low porosities $(<20 \%)$, followed by an increase illustrates the influence of the matrix anisotropy at low porosity and dominance of the crack anisotropy at high porosity. More complex scenarios can be further imagined, in which the lineation of the solid matrix is considered (i.e., an orthorhombic symmetry), as for cracks oriented obliquely to the principal axes of the solid matrix.

[13] To demonstrate the influence of aligned cracks on the $P$ wave anisotropy, as well as on the shear wave splitting and polarization of the "fast" propagating shear wave $\left(V_{S 1}\right)$, Figure 6 shows one scenario considering the single crystals muscovite and calcite, with $10 \%$ added cracklike porosity $(\alpha=$ 10). In this case the long axes of the cracks are aligned either parallel to bedding (or the basal plane of the crystal; Figures 6a and $6 \mathrm{c}$ ) or normal to bedding (crystal basal plane; Figures $6 \mathrm{~b}$ and $6 \mathrm{~d}$ ). The $V_{P}$ anisotropy is strongly reinforced by cracks aligned parallel to the bedding/basal plane of the crystals; whereas cracks oriented normal to the bedding/basal plane produce a more complex anisotropy. The total anisotropy decreases in the case of cracks oriented normal to the bedding/ basal plane of the crystal, but is higher than cases for single crystals without the presence of cracks. The $V_{S 1}$ polarization is also considerably different when cracks are oriented parallel compared to normal with the crystal bedding/basal plane. In the case of Figures $6 \mathrm{~b}$ and $6 \mathrm{~d}$, the polarization of a shear wave is controlled by the bedding/crystal basal plane when it propagates parallel or subparallel to the $X-Y$ plane (bedding plane). In contrast, the aligned cracks control the polarization for a shear wave propagating at a high angle to the bedding/basal plane (e.g., Figure $6 \mathrm{~b}$ ).

\section{Deducing Pore Shape Geometry Using Ferrofluids}

[14] As shown in section 2, the aspect ratio of pores and cracks has an important impact on the elastic properties of an effective medium. A small number of oriented flat cracks can, in fact, reduce the seismic velocities in a given direction of the medium by an amount that would require a much larger number of spherical pores. It is important to note that in natural geological settings idealized pore shape does not exist, and rather the pore space consists of a mixture of cracks and pores with irregular shapes. However, in order to be able to predict seismic velocities using effective medium theory it is necessary to assign specific and idealized ellipsoidal shape to the cracks and pores, providing a close approximation to the true shape of the porosity.

[15] There are several ways to obtain information on the pore space geometry. Often geometry is determined by thin section analysis. Here we consider a method which involves saturating the pore space with a ferrofluid by expelling the air from a sample under low vacuum conditions. A ferro- 

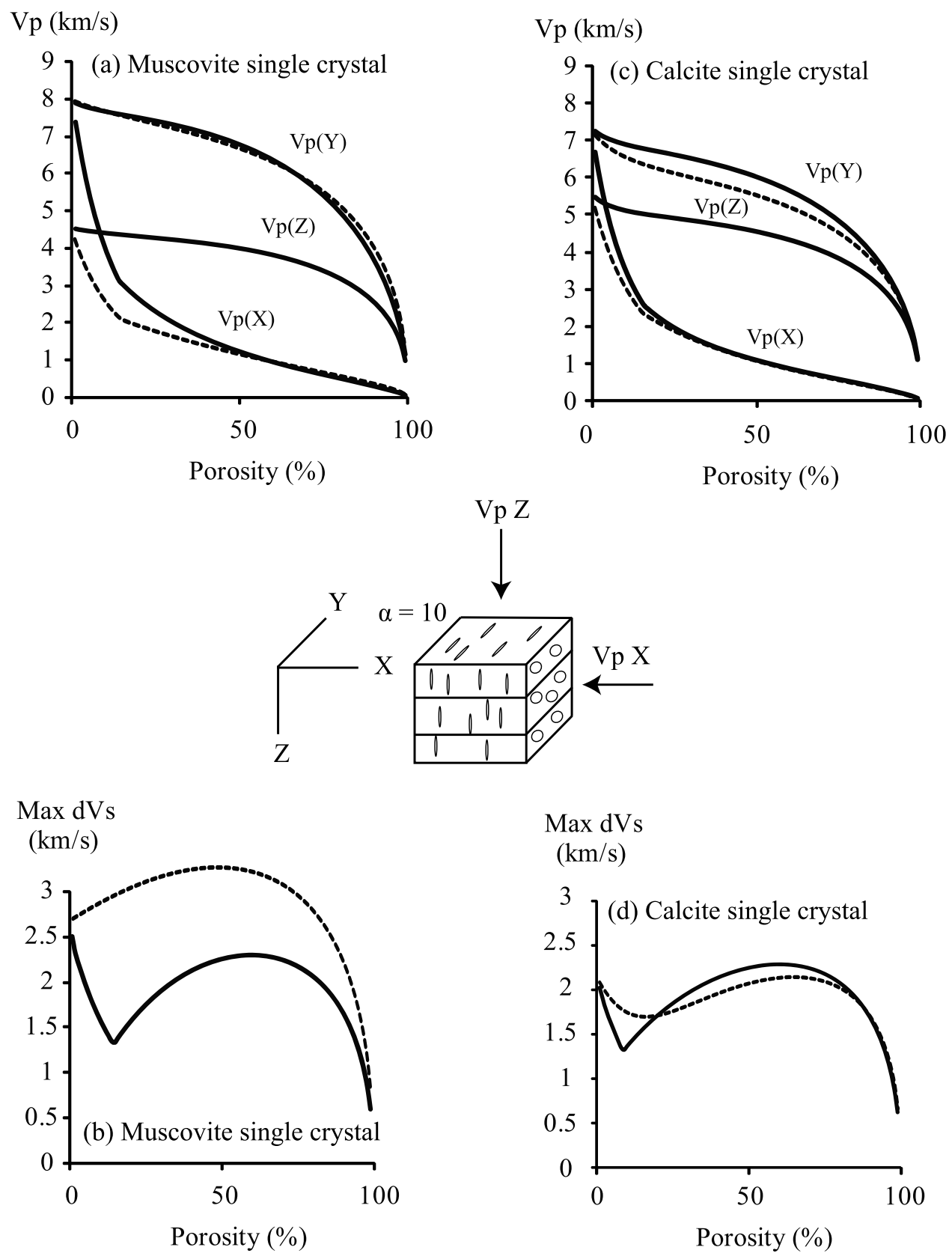

Figure 5. Seismic $P$ waves that propagate along sample $X, Y$, and $Z$ axes for single crystals of (a) muscovite and (c) calcite. Pores are oblate ellipsoids that have their short axis aligned along the sample $X$ axis; (b and d) the corresponding maximum shear wave splitting $\left(d V_{S}\right)$ for each of the single crystals. The dashed lines show the results from Figure 3 for the aspect ratio $\alpha=10$, as a comparison.

fluid is a colloidal suspension of very fine-grained particles of magnetite $(<20 \mathrm{~nm})$, whose magnetic moments are ideally noninteracting. The small grain size of the particles in the colloid makes them unable to carry a stable permanent magnetization, in contrast to magnetite of larger grain size. The particles have high magnetic susceptibility $(k)$, and their magnetization $(M)$ depends on the inducing magnetic field $(H)$, such that $M=k H$. In a weak applied field $(300 \mathrm{~A} / \mathrm{m}) k$ is a second rank symmetric tensor that relates $M$ and $H$, from which the anisotropy of magnetic susceptibility (AMS) for a sample can be determined. The eigenvalues and eigenvectors of the tensor provide the magnitude and directions of three principal axes $\left(k_{1} \geq k_{2} \geq k_{3}\right)$ of $k$, which can be represented geometrically by an ellipsoid. Hence, if a ferrofluid fills the pore space homogeneously, its AMS reflects pore shape or crack shape by an idealized ellipsoid. Typically the shape of the magnetic susceptibility ellipsoid and the degree of anisotropy is presented in magnetic fabric studies. The ellipsoid shape is defined as, $T=\left(2 \eta_{2}-\eta_{1}-\eta_{3}\right) /\left(\eta_{1}-\eta_{3}\right)$, where $\eta_{1}=\ln \left(k_{1}\right), \eta_{2}=\ln \left(k_{2}\right)$ and $\eta_{3}=\ln \left(k_{3}\right)$, for which the 

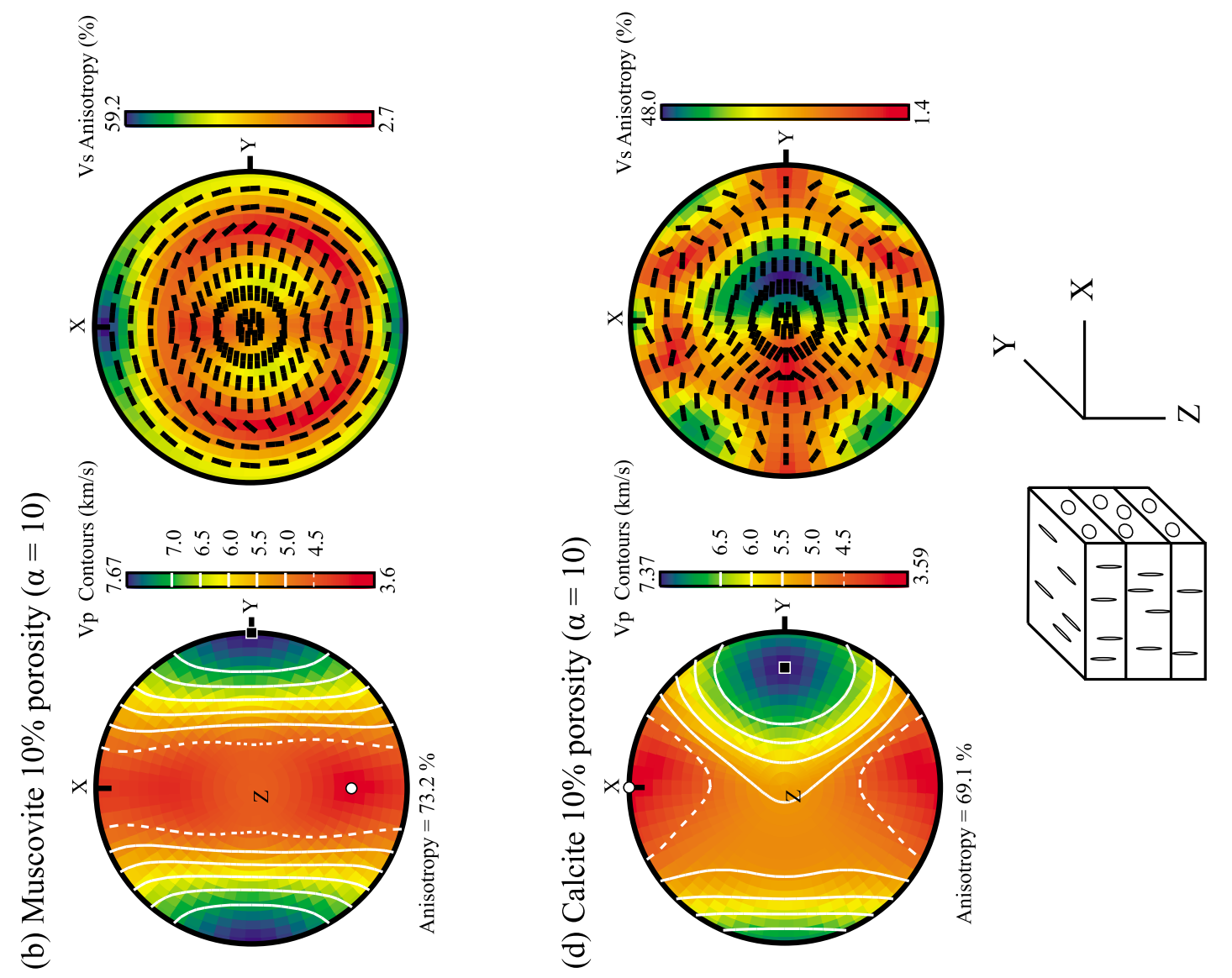

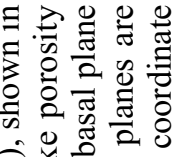
जि:

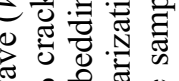
$3 \circ 0$ 웡

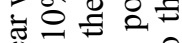

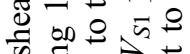

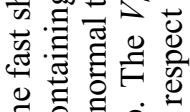
导 00 运

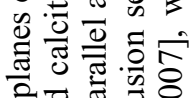
믈

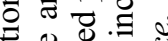
논. 흐을

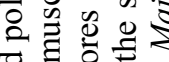
छ

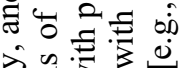
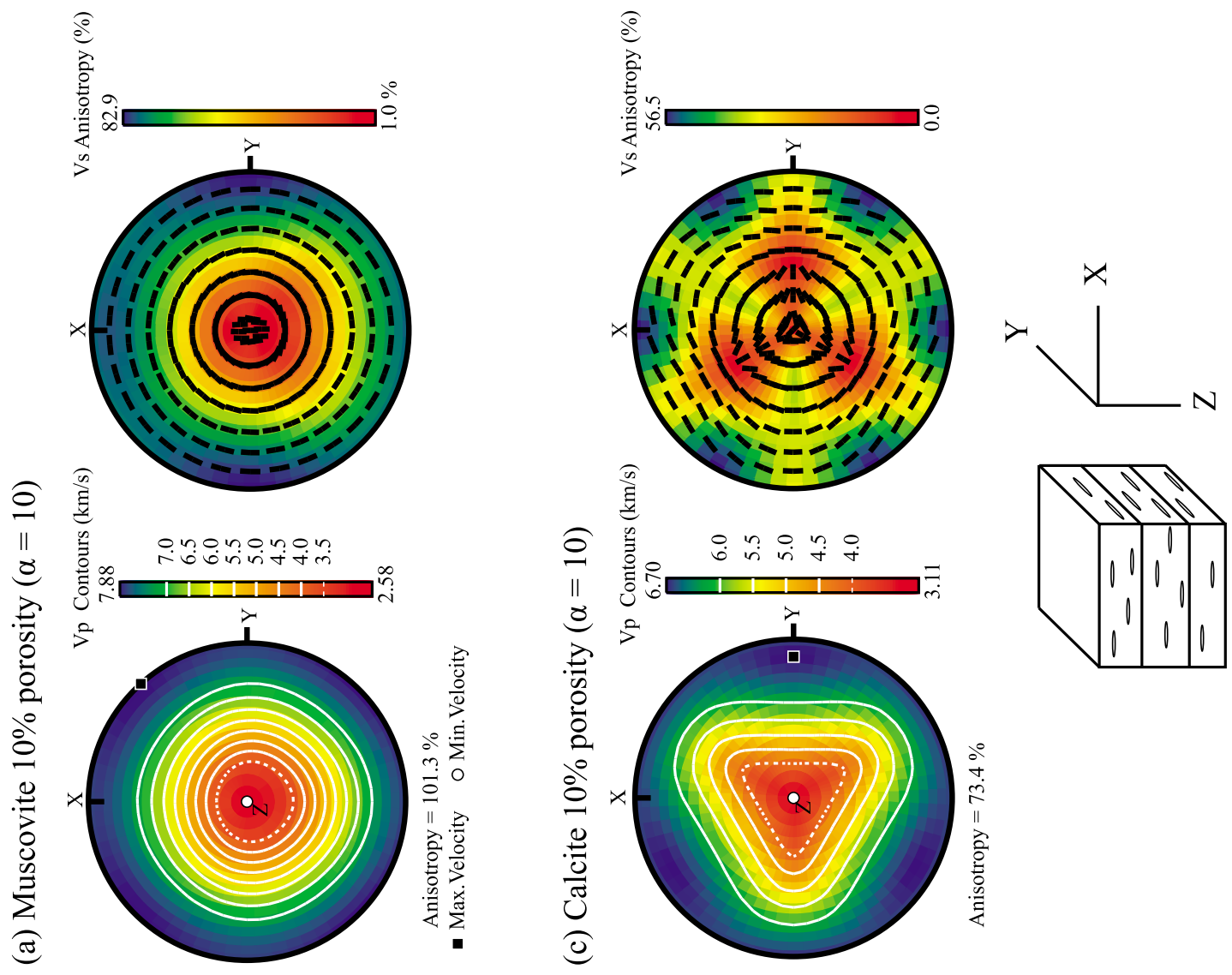

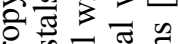

要焉造

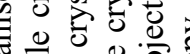

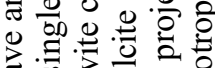

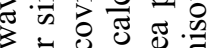

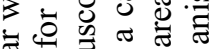
क छ की

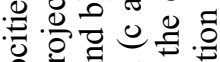

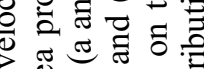

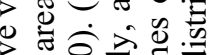
츠을 ३ ० क्ष 0 :

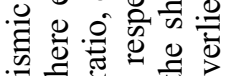
क की ن 论

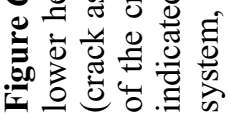


susceptibility ellipsoid is oblate shaped when $T>0$ to 1 , and prolate shaped when $T<0$ to -1 [Jelinek, 1981]. The degree of anisotropy $(P j)$ is a measure of the magnitude of the magnetic anisotropy and is defined as

$$
\ln (P j)=\sqrt{2}\left\{\left[\ln \left(\frac{k_{1}}{k}\right)\right]^{2}+\left[\ln \left(\frac{k_{2}}{k}\right)\right]^{2}+\left[\ln \left(\frac{k_{3}}{k}\right)\right]^{2}\right\}^{1 / 2} .
$$

The bulk susceptibility is defined as $k=\left(k_{1}+k_{2}+k_{3}\right) / 3$.

[16] The greatest obstacle faced when trying to relate the magnitude of the magnetic susceptibility principal axes to the actual pore shape arises from the demagnetizing factors of magnetic particles in the ferrofluid. A particle which is uniformly magnetized actually has a lower measured susceptibility than would be expected if the origin of the susceptibility was strictly external (or by surface poles), due to an internal magnetic field which acts in opposition to the external part of the particle's magnetization [Osborn, 1945; Stoner, 1945]. To overcome this problem, Hrouda et al. [2000] suggested using the equivalent pore concept (EPC), which is based on the work done by Stacey [1960] to determine the true axial ratio of magnetic particles. In principle, the EPC attempts to remove the effect that arise from the demagnetizing factors on the measured $k$, in order to obtain the principal axes of intrinsic susceptibility $\left(k_{i}\right)$, whose values provides an estimation of the bulk pore shape. The measured bulk susceptibility $(k)$ is related to the intrinsic susceptibility such that

$$
k=k_{i} /\left(1+N k_{i}\right)
$$

where $N$ is the demagnetizing factor. In the case of a sphere, the $N$ is $1 / 3$ [Uyeda et al., 1963]. The EPC can be applied with little effort in the case of an undiluted ferrofluid with a susceptibility less than 3 SI [e.g., Hrouda et al., 2000; Benson et al., 2003; Jones et al., 2006], but the large susceptibility carried by a pure ferrofluid can result in instrumental problems, as the instrument may become saturated by the strong ferrofluid signal. For this reason it may be preferable to measure a ferrofluid that has been diluted, or samples that have smaller size. Jones et al. [2006] showed that due to the variation in intrinsic susceptibility with various dilutions (fraction host medium to ferromagnetic particles) it becomes necessary to use a correction for the concentration effect when applying the EPC. However, in order to avoid a correction factor, the sample size was reduced rather than diluting the ferrofluid. From the intrinsic susceptibility of the ferrofluid an EPC chart can be computed specific to the ferrofluid. For this purpose we adopt a MATLAB routine written by Jezek and Hrouda [2007] to calculate the EPC based on the intrinsic susceptibility of the undiluted ferrofluid used in our experiments (MATLAB routine SUE.m is available through http://earthref.org/databases/ ERDA/).

[17] Despite the experimental challenges presented above, using the EPC is a viable alternative to obtain bulk estimate for the geometry of the pore space. The EPC is less time consuming than alternative methods (e.g., image autocorrelation with thin sections, $\mathrm{Hg}$ injection, and tomographic methods) and directly provides an ellipsoidal form for the pore shape required for DEM modeling.

\section{Elastic Moduli of Porous, Anisotropic Calcite- Muscovite Aggregates}

[18] Schmidt et al. [2008] described the procedure for the fabrication of a set of synthetic aggregates of calcite and muscovite. These mixtures were compacted with varying uniaxial loads, ranging from 20 to $400 \mathrm{MPa}$, to produce samples with different texture strengths. Each sample was afterwards hydrostatically pressed at a confining pressure of $150 \mathrm{MPa}$ and a temperature of $670^{\circ} \mathrm{C}$. The crystallographic preferred orientation (CPO), which is used for the seismic velocity calculations, was measured with neutron diffraction [Schmidt et al., 2009]. Seismic velocities were measured using a Paterson gas medium apparatus specifically equipped for ultrasonic measurements [cf. Almqvist et al., 2010, Table 1].

\subsection{Ferrofluid Measurements}

[19] Samples were impregnated with an oil-based ferrofluid, EMG 905, in its undiluted state. The synthetic samples were impregnated in a vacuum of approximately $10^{-3}$ bar for about $20 \mathrm{~h}$. The degree to which the ferrofluid saturated the samples was determined by measuring the initial mass of an oven-dried specimen, and comparing it with the mass after impregnation with the ferrofluid. Using the density of the fluid, it was possible to calculate the amount of fluid entering the sample (Table 1). After sample impregnation the anisotropy of magnetic susceptibility (AMS) was measured [e.g., Pfleiderer and Halls, 1990, 1994; Benson et al., 2003; Louis et al., 2005]. The AMS reflects the amount and distribution of the ferrofluid, which has filled pores and cracks in the sample, since the fluid carry strongly magnetic particles in comparison to the weakly paramagnetic and diamagnetic mineral susceptibilities of muscovite and calcite. The bulk susceptibility of fluid-filled specimens is three orders of magnitude or higher than the samples in a dry state (Table 1). It must be noted that this method provides a measure of the bulk pore/crack anisotropy, which is an average of the individual pores and cracks. It is therefore not possible to express the possible range of crack and pore geometries in each sample. Nevertheless, a seismic wave will experience the integrated effect of the network of cracks and pores in the rock, and in such a way experience the average geometry of the pore/crack network.

[20] AMS measurements, subsequent to impregnation with the ferrofluid, display a consistent magnetic fabric for all specimens where the $\mathrm{k}_{3}$ axis is normal to the foliation plane and the $k_{1}$ and $k_{2}$ axes are dispersed in the foliation plane, producing of a planar magnetic fabric. With the exception of samples with 5\% muscovite, the AMS ellipsoids are close to rotational oblate shape, indicating that the porosity has a transversely isotropic symmetry for all samples, with the symmetry axis normal to the foliation plane (Figure 7a). The amount of ferrofluid saturation, as inferred from the bulk susceptibility (Figure 7b), depends on the sample composition and the load used to compact the samples. To a lesser extent the ferrofluid also depends on the initial sample porosity, which was highest for $70 \%$ muscovite content with a porosity of $20-25 \%$. The highest bulk susceptibility is recorded for samples with $50 \%$ calcite and 
Table 1. Summary of Sample Properties and AMS Measurements After Ferrofluid Impregnation

\begin{tabular}{|c|c|c|c|c|c|c|c|c|c|c|}
\hline Sample & $\begin{array}{c}\text { Muscovite } \\
(\%)\end{array}$ & $\begin{array}{l}\text { Uniaxial Load } \\
\text { (MPa) }\end{array}$ & $\begin{array}{l}\text { Porosity } \\
(\%)\end{array}$ & $\begin{array}{l}\text { Sample Mass } \\
\text { (g) }\end{array}$ & $\begin{array}{l}\text { Ferrofluid Mass } \\
\text { (g) }\end{array}$ & $k\left(\times 10^{-6} \mathrm{SI}\right)$ & $P j$ & $T$ & $a / b^{\mathrm{a}}$ & $b / c^{\mathrm{a}}$ \\
\hline $0-20$ & 0 & 20 & 3.7 & 6.1047 & 0.0592 & 1996 & 1.061 & 0.91 & 1.004 & 1.089 \\
\hline $0-100$ & 0 & 100 & 3.5 & 6.3351 & 0.0584 & 2487 & 1.089 & 0.98 & 1.001 & 1.135 \\
\hline $5-40$ & 5 & 40 & 5.4 & 6.3645 & 0.1035 & 2763 & 1.067 & 0.98 & 1.001 & 1.101 \\
\hline $5-200$ & 5 & 200 & 6.3 & 6.3455 & 0.0845 & 1951 & 1.070 & 0.92 & 1.004 & 1.103 \\
\hline $5-400$ & 5 & 400 & 5 & 6.1788 & 0.0782 & 1550 & 1.082 & 0.96 & 1.002 & 1.123 \\
\hline $10-100$ & 10 & 100 & 7.5 & 5.9340 & 0.1376 & 4898 & 1.090 & 0.93 & 1.005 & 1.133 \\
\hline $30-20-2$ & 30 & 20 & 15 & 5.5727 & 0.3358 & 31485 & 1.132 & 0.92 & 1.008 & 1.196 \\
\hline $30-100-2$ & 30 & 100 & 13.5 & 5.7808 & 0.2884 & 25081 & 1.149 & 0.91 & 1.010 & 1.221 \\
\hline $30-200-2$ & 30 & 200 & 12.2 & 5.9407 & 0.2554 & 17874 & 1.156 & 0.97 & 1.003 & 1.239 \\
\hline $50-20-2$ & 50 & 20 & 18 & 5.6182 & 0.4724 & 46083 & 1.174 & 0.97 & 1.004 & 1.268 \\
\hline $50-20-\mathrm{s}$ & 50 & 20 & 22.6 & 5.0417 & 0.4984 & 37974 & 1.099 & 0.99 & 1.001 & 1.152 \\
\hline $50-100-2$ & 50 & 100 & 16.7 & 5.5703 & 0.3938 & 37193 & 1.184 & 0.95 & 1.007 & 1.281 \\
\hline $50-100-\mathrm{s}$ & 50 & 100 & 20 & 5.1147 & 0.454 & 35657 & 1.141 & 0.98 & 1.002 & 1.217 \\
\hline $50-200-2$ & 50 & 200 & 16 & 5.6337 & 0.3511 & 30597 & 1.220 & 0.98 & 1.004 & 1.344 \\
\hline $50-200-\mathrm{s}$ & 50 & 200 & 19 & 5.6027 & 0.4342 & 29005 & 1.139 & 0.98 & 1.002 & 1.214 \\
\hline $70-20$ & 70 & 20 & 24.5 & 5.1419 & 0.4667 & 31073 & 1.179 & 0.92 & 1.010 & 1.268 \\
\hline $70-100$ & 70 & 100 & 21.5 & 5.5008 & 0.4592 & 24227 & 1.237 & 0.94 & 1.010 & 1.364 \\
\hline $70-200$ & 70 & 200 & 19.7 & 5.5675 & 0.3946 & 23318 & 1.258 & 0.84 & 1.029 & 1.373 \\
\hline $100-20$ & 100 & 20 & 14.1 & 5.5445 & 0.236 & 3319 & 1.222 & 0.97 & 1.005 & 1.346 \\
\hline $100-100$ & 100 & 100 & 12.1 & 6.1892 & 0.2208 & 2142 & 1.260 & 0.95 & 1.009 & 1.403 \\
\hline $100-200$ & 100 & 200 & 11.9 & 5.6637 & 0.1936 & 1374 & 1.249 & 0.97 & 1.006 & 1.390 \\
\hline
\end{tabular}

${ }^{\mathrm{a}}$ Axial ratios determined with the EPC.

$50 \%$ muscovite. Samples with pure muscovite composition display similar bulk susceptibilities as pure calcite samples, even though the porosity is $12-14 \%$ in the former and $3.5-$ $4 \%$ in the latter. In contrast, the degree of anisotropy $(P j)$ is higher with increasing muscovite content, as well as with increasing volume of pores (Figure 7c). It can be inferred therefore that the shape of pores is dependent on the amount of muscovite and the compaction load. The AMS shape ellipsoids are nearly rotational oblate for the entire group of samples (Table 1). Based on the intrinsic susceptibility of the EMG 905 ferrofluid, 3.707 SI [Hrouda et al., 2000], it is possible to employ the EPC to compute the actual shape of the pores filled by the ferrofluid. The results of the EPC are shown in Figure 7d, indicating that pores have an aspect ratio $\alpha<2$ for all samples, and are nearly rotational oblate $(\mathrm{a} \sim \mathrm{b}>\mathrm{c})$.

\subsection{Aggregate Effective Elastic Properties}

[21] Elastic properties are first calculated for the solid matrix, as described above, using the single-crystal elastic properties, the calcite and muscovite volume fractions, and CPO obtained from neutron diffraction measurements. The results for the modeled solid matrix have been presented in Almqvist et al. [2010, Table 1]. Not surprisingly the samples that have high muscovite content and compacted with a large uniaxial load display a larger anisotropy compared to samples with high calcite content, and a small applied uniaxial load. The highest $V_{P}$ are found in the plane perpendicular to the symmetry axis, which is also the compression axis; this plane also produces the largest velocity differences between the fast and slow shear waves $\left(V_{S 1}\right.$ and $V_{S 2}$ ), that is, maximum shear wave splitting. A comparison of measured and predicted seismic velocities, without considering porosity, is shown in Figure 8a. Predicted velocities are always higher than those obtained from measurements as expected, because of the absence of pores in the model.
[22] The elastic properties for the effective medium are calculated by considering porosity and pore shape using the DEM. Porosity, in general, increases with higher muscovite to calcite ratio, with the notable exception of pure muscovite samples. The pore shape, deduced from AMS measurements of the ferrofluid-filled samples, is nearly spherical but varies slightly depending on sample compaction load and composition (Table 1). The known porosity and pore axial ratios are used as input parameters for the DEM, and the porefilling medium is air, which is assigned elastic moduli of $0 \mathrm{GPa}$ (strictly the adiabatic bulk modulus of dry air is $1.42 \times$ $10^{-4} \mathrm{GPa}$ ), and a density of $1.2 \mathrm{~kg} / \mathrm{m}^{3}$. The combined calculations for the solid matrix and the incorporation of dry pores with the DEM provide the complete anisotropic 4thrank elastic tensor. The $V_{P}$ and $V_{S}$ derived from the DEM moduli are presented in Table 2 and compared with measured $V_{P}$ and $V_{S}$ (at $300 \mathrm{MPa}$ ) in Figure $8 \mathrm{~b}$. Note that the measured velocities listed in Figure 8 were obtained at $300 \mathrm{MPa}$ confining pressure, and the porosity has decreased slightly during measurements, which is assumed based on increasing velocities at higher confining pressure, but these measurements provide a first order comparison with the DEM computed velocities. The porosity used in the DEM calculations is the porosity that is measured subsequent to the experimental run, in order to get as close to the actual porosity at a confining pressure of $300 \mathrm{MPa}$. As seen in Table 1, the permanent reduction in porosity after the experiment is generally between 1 and $2 \%$, differing little with sample composition. In comparison, an overall aspect ratio for the group of samples that best matches the measured seismic velocities is between $\alpha=3$ and $\alpha=4$ (Figure 8c) [Almqvist et al., 2010].

\section{X-Ray Microtomography}

[23] In order to independently investigate the ferrofluid impregnation we have used X-ray computed tomography. The system we used consists of a SkyScan 1172 high- 

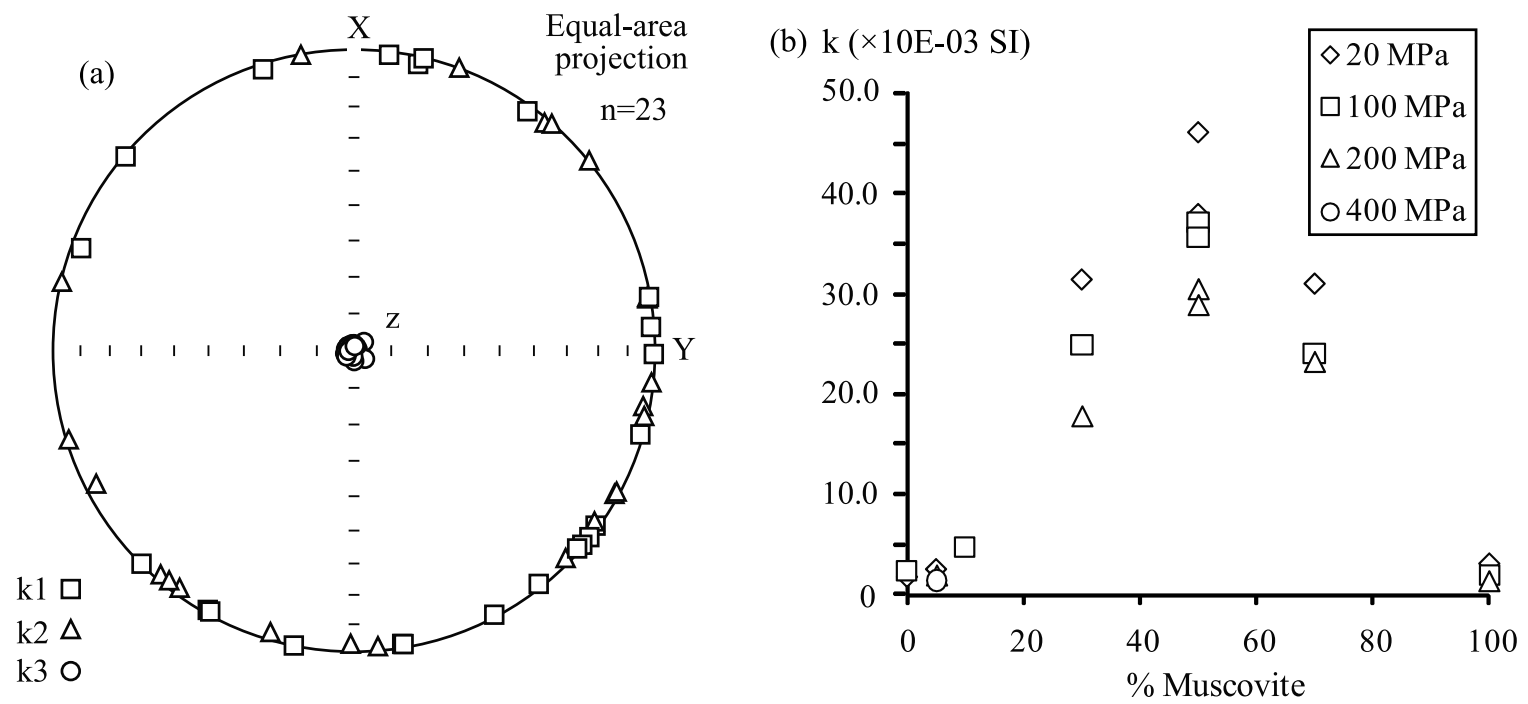

(c) $\mathrm{Pj}$

(d) $\mathrm{a} / \mathrm{c}$
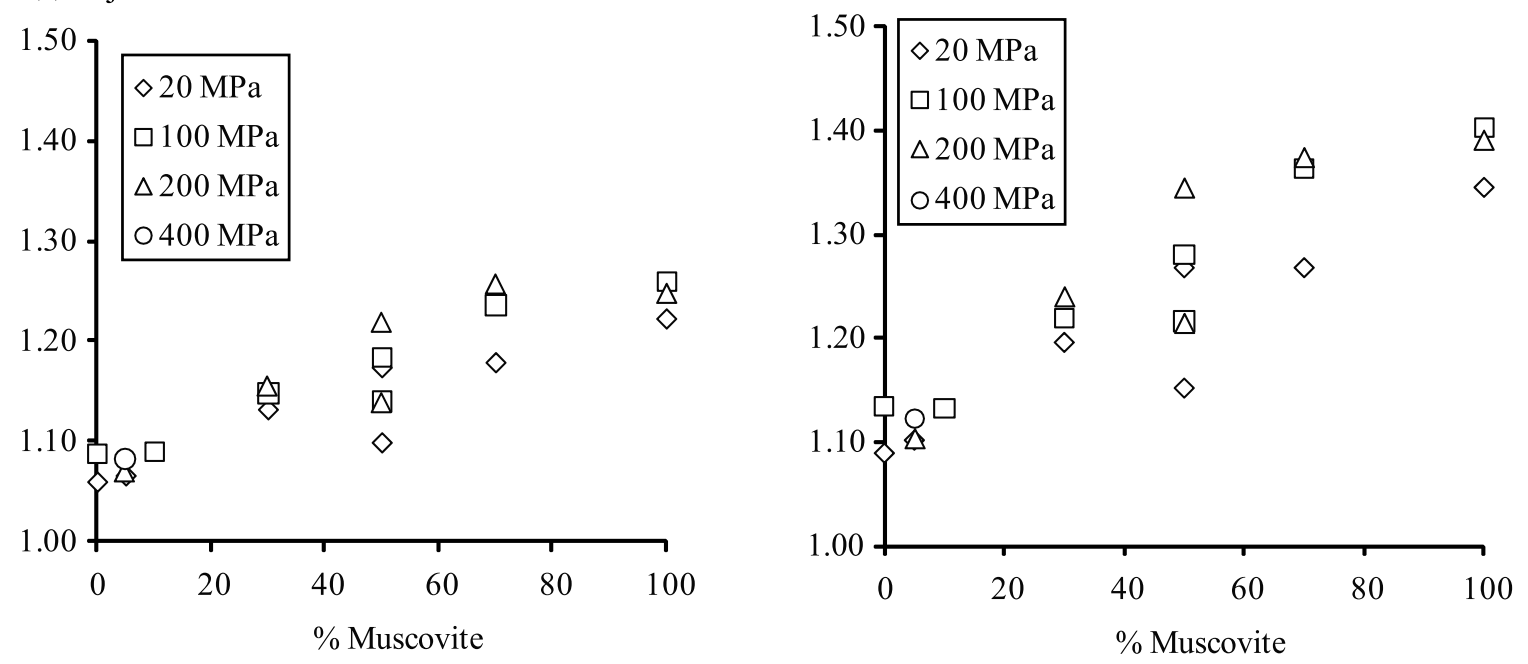

Figure 7. Results from the ferrofluid impregnation experiments. (a) Equal area projection with axes of maximum $\left(k_{1}\right)$, intermediate $\left(k_{2}\right)$ and minimum $\left(k_{3}\right)$ susceptibility; $\mathrm{X}, \mathrm{Y}$ and $\mathrm{Z}$ refer to the sample coordinate system described in the text and in Figure 3. (b) Bulk susceptibility as a function of sample composition, for various uniaxial loads used during sample manufacturing. (c) The degree of anisotropy $(P j)$ as a function of sample composition, for various uniaxial loads used during sample manufacturing. (d) The application of the equivalent pore concept (EPC), showing the aspect ratio of pores, derived from the AMS measurements of the ferrofluid-filled samples, as a function of sample mineral composition and uniaxial load used during sample manufacturing.

resolution desktop scanner (SkyScan NV, Kontich, Belgium), equipped with a $100 \mathrm{kV}$ X-ray source and a 10 Megapixel CCD camera. The attenuation of X-rays is related to the density contrast of the material. Therefore, we can compare the tomography measurements performed on a dry sample and a ferrofluid saturated sample. Two cores of $6 \mathrm{~mm}$ diameter and $4 \mathrm{~mm}$ length from the same sample were used for the measurements. The samples had a composition of $70 \mathrm{vol} \%$ muscovite and $30 \mathrm{vol} \%$ calcite, and have been compacted with $20 \mathrm{MPa}$ uniaxial load. The initial porosity of this sample is $24.5 \%$.

[24] The samples were scanned with a tube voltage of $81 \mathrm{kV}$, a current of $109 \mu \mathrm{A}$, a $0.5 \mathrm{~mm} \mathrm{Al}$ filter, and the rotation step size was $0.31^{\circ}$ at a full $360^{\circ}$ rotation. At these operating conditions the resulting pixel size was $1.95 \mu \mathrm{m}$. Transmission images were reconstructed using SkyScan's NRecon cluster software v.1.6.1. The artifact correction parameters were set to 6 (ring artifact correction) and $32 \%$ (beam-hardening correction). For each core, the complete data set is composed of 1400 cross sections.

[25] Figures 9a and 9b illustrate two cross sections of the raw data for the dry and ferrofluid saturated cores. In general the pore size is small $(<100 \mu \mathrm{m})$ and homogeneously distributed (i.e., no heterogeneity in pore size or shape through the sample due to the sample preparation). After impregnation there was a clear visual difference in the bulk 

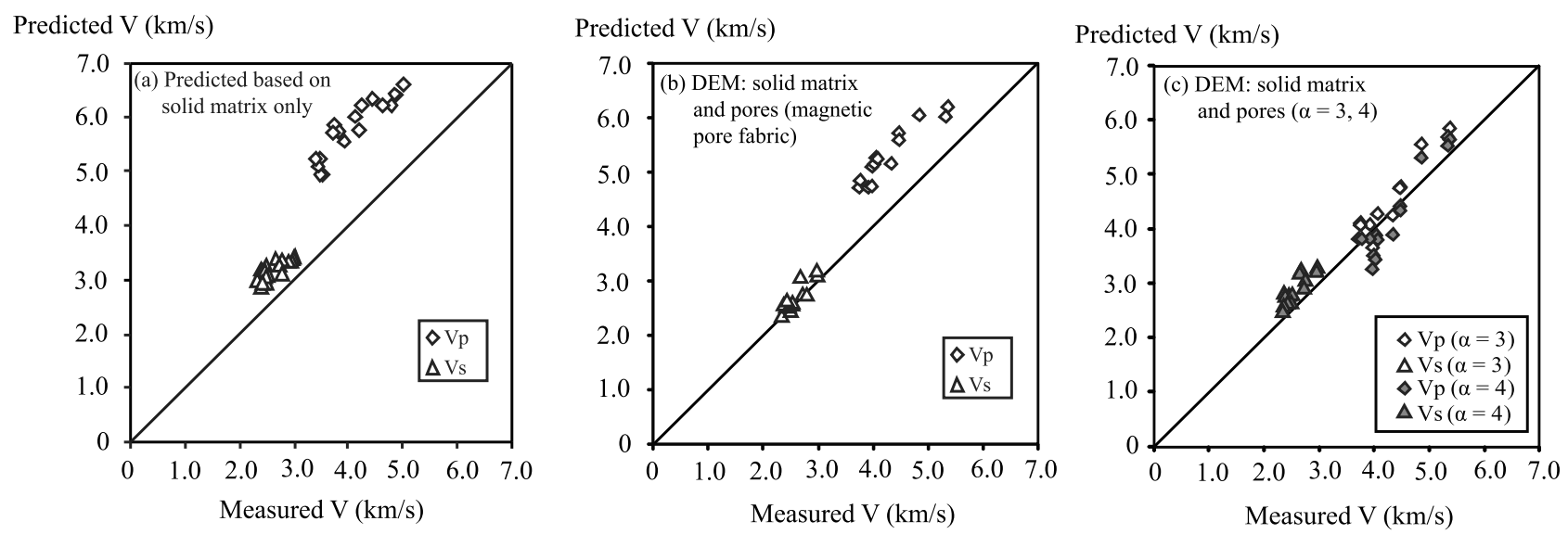

Figure 8. Predicted compressional wave and shear wave velocities are shown with respect to measured velocities based on (a) the solid matrix and (b) the solid matrix plus inclusion of pores that have aspect ratios based on the EPC. (c) Predicted $P$ and $S$ wave velocities when $\alpha=3$ and $\alpha=4$.

absorption between the cores. Most of the ferrofluid is located in the rim of the sample, although, some of the fluid appears to have migrated to the center of the core. At present we must evaluate whether this is due to real ferrofluid distribution or, alternatively, due to uncorrected beam hardening.

[26] To quantitatively compare the dry and saturated sample we calculated the attenuation coefficient, which provides an indication of the bulk material density (Figure 9c). Comparatively, the ferrofluid-saturated sample displays a higher bulk attenuation coefficient, which indicates that the sample has an overall higher density. Since both cores have been scanned and reconstructed under identical conditions, we can largely attribute the increase in X-ray attenuation to the ferrofluid.

[27] A volume of the dry sample, whose location is indicated by the shaded orange square in Figure 9a, has been investigated to determine the amount, shape, and interconnectedness of pores. For this purpose a model was constructed with CT-analyzer v.1.10 software. The scanned data was binarized as representing either solid matrix or open pores, using an appropriate threshold value based on the collective gray scale histogram brightness distribution. Images of the processed data from this sample volume are shown in Figures 9d and 9e, which illustrate the distribution of pores in black and by shading, respectively. The processed volume of the specimen yields a total porosity of $24.9 \%$, which is in good agreement with the porosity listed in Table 1; more than $99 \%$ of the pores are interconnected. Based on the total volume of pores and number of objects identified from the data processing, the estimated average pore size is $\sim 20 \mu \mathrm{m}$. The volume averaged aspect ratio of pores, that is, the degree of anisotropy, $P j$, is 2.16 . This value is somewhat larger than that derived from the ferrofluid measurements, which was 1.27 (Table 1), but is close to the best fit average pore shape determined by Almqvist et al. [2010].

\section{Discussion}

\subsection{Modeling the Effective Medium}

[28] A DEM model has been applied to predict seismic velocities in porous aggregates consisting of compacted

Table 2. Predicted Seismic Velocities Obtained With the DEM

\begin{tabular}{|c|c|c|c|c|c|c|c|c|c|c|c|c|c|}
\hline Sample & $\begin{array}{c}\text { Muscovite } \\
(\%)\end{array}$ & $\begin{array}{l}\text { Compaction Load } \\
(\mathrm{MPa})\end{array}$ & $\varphi^{\mathrm{a}}(\%)$ & $\rho^{\mathrm{b}}\left(\mathrm{g} / \mathrm{cm}^{3}\right)$ & $V_{P h}{ }^{\mathrm{c}}$ & $V_{S h}{ }^{\mathrm{c}}$ & $V_{s v}{ }^{\mathrm{c}}$ & $V_{P v}{ }^{\mathrm{c}}$ & $V_{S v, v h}{ }^{\mathrm{c}}$ & $A V_{p}(\%)$ & Maximum $d V s$ & $K^{\mathrm{d}}$ & $G^{\mathrm{d}}$ \\
\hline $5-40-2$ & 5 & 40 & 4.5 & 2.59 & 6.52 & 3.48 & 3.37 & 6.23 & 3.37 & 4.7 & 0.12 & 66.84 & 30.10 \\
\hline $5-200-2$ & 5 & 200 & 4.1 & 2.60 & 6.69 & 3.59 & 3.32 & 6.02 & 3.32 & 10.7 & 0.28 & 68.43 & 30.29 \\
\hline $10-100-2$ & 10 & 100 & 6.3 & 2.55 & 6.47 & 3.48 & 3.30 & 6.06 & 3.32 & 6.6 & 0.18 & 63.62 & 28.91 \\
\hline $30-20-2$ & 30 & 20 & 13.6 & 2.37 & 6.04 & 3.37 & 3.18 & 5.72 & 3.19 & 5.4 & 0.19 & 50.22 & 25.01 \\
\hline $30-100-2$ & 30 & 100 & 11.7 & 2.42 & 6.30 & 3.54 & 3.12 & 5.60 & 3.13 & 11.9 & 0.42 & 54.88 & 25.79 \\
\hline $50-20-2$ & 50 & 20 & 16.2 & 2.32 & 6.10 & 3.52 & 3.01 & 5.25 & 3.01 & 15.3 & 0.52 & 47.33 & 23.48 \\
\hline $50-20-\mathrm{s}$ & 50 & 20 & 15.6 & 2.34 & 5.79 & 3.35 & 2.98 & 5.15 & 2.98 & 11.7 & 0.37 & 48.57 & 23.62 \\
\hline $50-100-2$ & 50 & 100 & 14.4 & 2.37 & 6.13 & 3.53 & 3.00 & 5.28 & 3.00 & 15.3 & 0.53 & 52.50 & 23.96 \\
\hline $50-100-\mathrm{s}$ & 50 & 100 & 21.4 & 2.18 & 5.97 & 3.46 & 2.93 & 5.10 & 2.93 & 16.0 & 0.53 & 39.82 & 20.90 \\
\hline $50-200-2$ & 50 & 200 & 19.6 & 2.23 & 6.38 & 3.68 & 2.93 & 5.16 & 2.93 & 21.1 & 0.76 & 43.39 & 21.49 \\
\hline $70-100-r$ & 70 & 70 & 19.8 & 2.24 & 6.22 & 3.68 & 2.73 & 4.74 & 2.74 & 27.3 & 0.95 & 45.84 & 20.83 \\
\hline $100-20$ & 100 & 20 & 12.6 & 2.47 & 6.28 & 3.77 & 2.92 & 4.84 & 2.93 & 26.3 & 0.85 & 49.51 & 25.43 \\
\hline $100-100$ & 100 & 100 & 11.3 & 2.51 & 6.57 & 3.95 & 2.79 & 4.72 & 2.80 & 32.9 & 1.17 & 55.04 & 25.40 \\
\hline $100-200$ & 100 & 200 & 11.0 & 2.52 & 6.64 & 4.00 & 2.76 & 4.71 & 2.76 & 34.1 & 1.24 & 56.64 & 25.35 \\
\hline
\end{tabular}

\footnotetext{
${ }^{\text {a }}$ Porosity.

${ }^{\mathrm{b}}$ Density.

${ }^{\mathrm{c}}$ Velocities in $\mathrm{km} / \mathrm{s}$.

${ }^{\mathrm{d}}$ Average bulk and shear moduli for each aggregate (GPa).
} 
a) Sample 70-20 dry
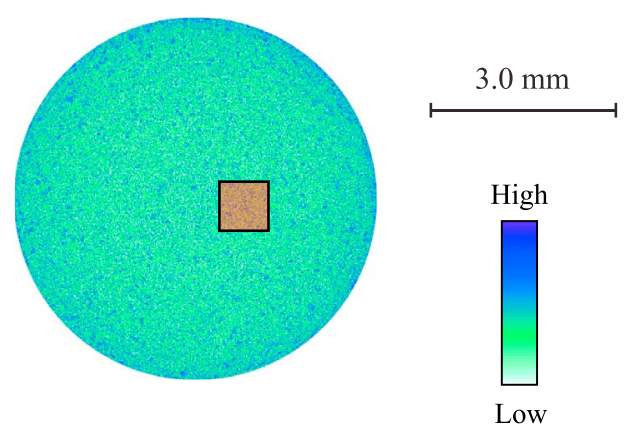

b) Sample 70-20 + ferrofluid

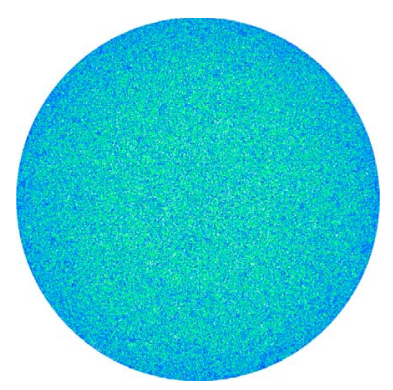

c) Intensity (a.u.)

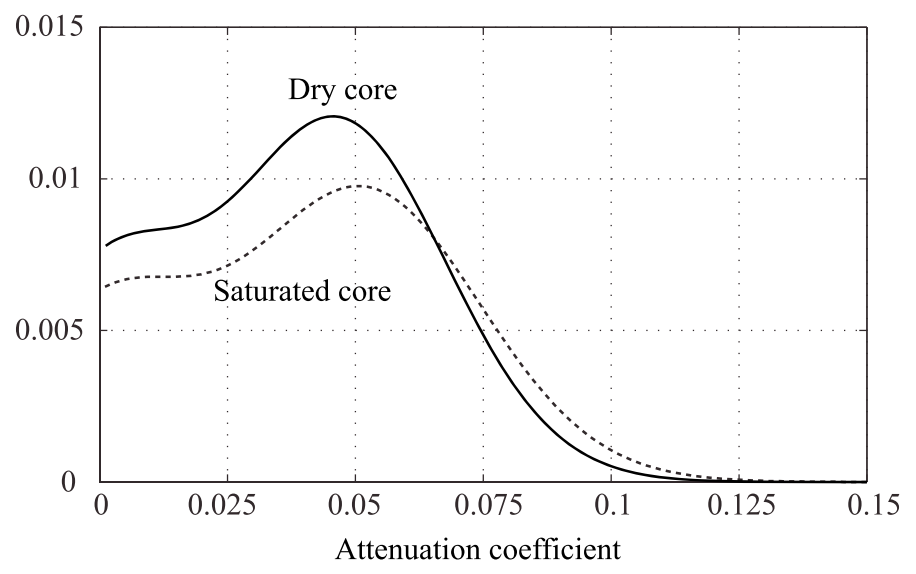

d)

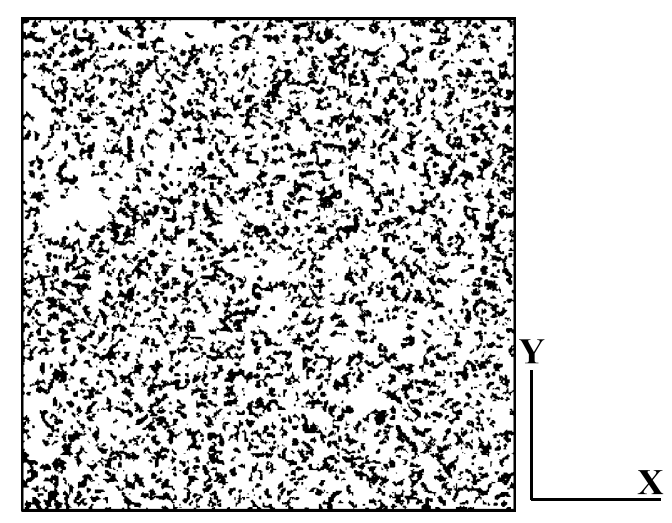

e)

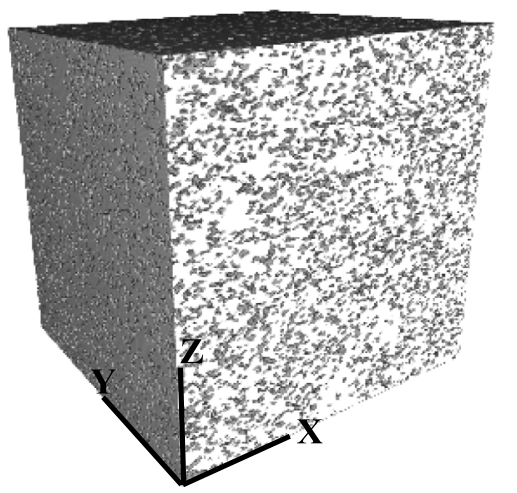

Figure 9. X-ray tomographic cross sections of (a) the dry core and (b) the ferrofluid-saturated core. The images show the plane of compaction. The color legend indicates high versus low attenuation of X-rays. (c) Intensity of attenuation shown as a function of the attenuation coefficient. Note that the curve of the ferrofluid-saturated core is shifted toward the right indicating that the attenuation is higher for this core. The shaded orange square inset in Figure 9a represents a volume of the specimen shown in Figures 9d and 9e (pores are black and shaded in Figures 9d and 9e, respectively, while the solid matrix is shown in white; the $Z$ axis represent the axis of compaction (symmetry axis) and $X$ and $Y$ axes lie in the plane of compaction).

mixtures of calcite and muscovite. Due to uniaxial compaction these aggregates have a transversely isotropic symmetry, as shown previously by texture measurements, anisotropy of magnetic susceptibility and acoustic velocity measurements [Schmidt et al., 2008, 2009; Almqvist et al., 2010]. The initial porosity was high after sample synthesis
(Table 1), and has a strong influence on the propagation of $P$ and $S$ waves. The DEM is able to account for an anisotropic background matrix and for inclusions of specific, idealized, ellipsoidal geometry. The average pore geometry has been inferred by filling the empty pores with a ferrofluid and measuring the anisotropy of magnetic susceptibility of these 
ferrofluid-impregnated samples. This study considers the case of a dry inclusion, since this is suitable to compare with high-frequency elastic wave measurements in the laboratory, whereas a fluid-saturated samples likely differences between measured and predicted velocities due to a high-frequency dispersion mechanism [see Le Ravalec and Guéguen, 1996].

[29] The compaction procedure of the synthetic samples suggests that the pores are shaped as flattened ellipsoids, with long axes in the plane normal to the axis of compaction. In the work by Almqvist et al. [2010] an attempt was made to find the pore shape that best explained the reduction in compressional and shear velocities, where several different aspect ratios are assigned to the pores. The agreement between experiment and model calculations is best for pore shapes between 3:3:1 and 4:4:1 (Figure 8c), suggesting that this is closest to the volumetrically averaged aspect ratio of the pores sampled by the ultrasonic elastic waves when the complete sample set is considered. However, the volume averaged aspect ratio is higher than indicated by ferrofluid impregnation and X-ray microtomography. Several effects influence the shape of the pores in the synthetic aggregates, including a mixture of grains with platy and euhedral character (muscovite and calcite), several different compositions, and also the different uniaxial loads used during synthesis.

[30] Prediction of the seismic velocities with the DEM, using the axial ratios of pores based on the EPC, do not fully explain the low measured seismic velocities in the case of compressional waves, but do agree with the measured shear wave velocities, although these are clearly less sensitive to porosity. Two reasons are likely for the underestimation of the modeled velocities: (1) The ferrofluid has not fully saturated the samples, and the porous spaces that are filled are biased toward spherical pores; and (2) microstructural properties that are not incorporated in the DEM are responsible for lowering the compressional wave velocities, such as velocity reduction due to the granular framework [e.g., Almqvist et al., 2010; Wenk et al., 2008; Voltolini et al., 2009], which is a likely reason for the overestimation of the predicted best fit aspect ratio shown in Figure 8c.

\subsection{Ferrofluid Transport Properties}

[31] The pores of the specimens are not completely filled with the ferrofluid during vacuum conditions. Two questions arise with regards to the ferrofluid experiments. First, why are specimens not completely saturated by the fluid? Second, where in the sample is the ferrofluid hosted? In addition, the bulk susceptibility indicates that there are compositional and compaction effects, which inhibit ferrofluid saturation, particularly when the muscovite content is high $(>50 \%)$. It is therefore important to address the question as to whether some pores are filled preferentially to others (and why this occurs) in order to validate the use of ferrofluids as a proxy for pore shape. Previous work by Hrouda et al. [2000] and Jones et al. [2006] illustrated that the use of ferrofluids to saturate ceramics and polycarbonate cylinders with known pore aspect ratios and pore geometry could be used successfully to recreate the actual pore shape of the specimen. However, the work by Jones et al. [2006] considered specimens with large pores (millimeter scale), which the ferrofluid could easily fill during their experiments. In the work of Hrouda et al. [2000] the pore size was not specified.
Pfleiderer and Kissel [1994] showed for a set of siltstones that the amount of ferrofluid that entered the samples during impregnation is directly related to the bulk susceptibility. They were able to successfully impregnate the siltstones with ferrofluid despite that the permeability of these rocks sometimes can be as low as $10^{-16}-10^{-17} \mathrm{~m}^{2}$. Permeability on this order and higher includes a large portion of rocks, with the exclusion of rocks hosting minerals that reduce permeability such as fine-grained shale and chalks [Mallon et al., 2005], and rocks with intrinsic low permeability such as fresh granites. Since impregnation of the ferrofluid was performed over the same time span for each sample $(\sim 20 \mathrm{~h})$, the amount of ferrofluid in the samples and the sample porosity can be used to provide insight on the relative permeability, or transport properties of the ferrofluid, in the group of synthetic samples (Figure 10). From the data in Figure 10 it is possible to infer samples which are easy to impregnate relative to their total porosity, since all samples were impregnated with the ferrofluid under similar conditions. For example, samples that contain 30 and $50 \%$ muscovite have large bulk susceptibilities after ferrofluid impregnation. However, the highest total porosity belongs to samples containing $70 \%$ muscovite. The composition therefore plays an important role for the permeability of the samples, where high muscovite content reduces permeability significantly. This is seen particularly for samples containing $100 \%$ muscovite, where the bulk susceptibility is very low $\left(1370 \times 10^{-6}\right.$ to $3320 \times 10^{-6} \mathrm{SI}$; Table 2), relative to their total porosity. Compaction of the pure mica samples, which consist of thin platy minerals, leads to a less permeable network, compared with mixtures of calcite and muscovite. The compaction load used during synthesis is the second factor that influences sample permeability. Samples that were compacted with large loads (100 and $200 \mathrm{MPa}$ ) have consistently lower bulk susceptibilities than samples compacted with small loads (20 MPa).

[32] From the observations above this implies that the amount of ferrofluid that impregnated the samples depends on the composition of the specimens and the load used to compact specimens during synthesis. The first effect can partly be explained by the large difference in shape between calcite and muscovite. The compaction of increasing amounts of muscovite, being a thin platy mineral, decreases the size and aspect ratio of the pores and pore throats, and reduces the permeability of the specimen. The degree of anisotropy increases with high muscovite concentrations and this may influence the ease with which the ferrofluid is able to flow through the pore network, as reflected by the lower bulk susceptibility of specimens with 70 and $100 \%$ muscovite composition. The increasing compaction load used during synthesis also appears to inhibit the flow of ferrofluid during vacuum conditions, although this effect is less readily identified, since the initial porosity also decreases with increasing compaction load. The ferrofluid flows most easily through large pores and cavities, and occupy pores with near spherical aspect ratio, whereas the fluid is restricted to enter narrow pores with small aspect ratios during imbibition. When measuring the AMS this leads to a bias toward pores with aspect ratios that are near spherical, which helps explain why aspect ratios predicted by the EPC does not fully explain measured seismic velocities to those predicted by the DEM. 

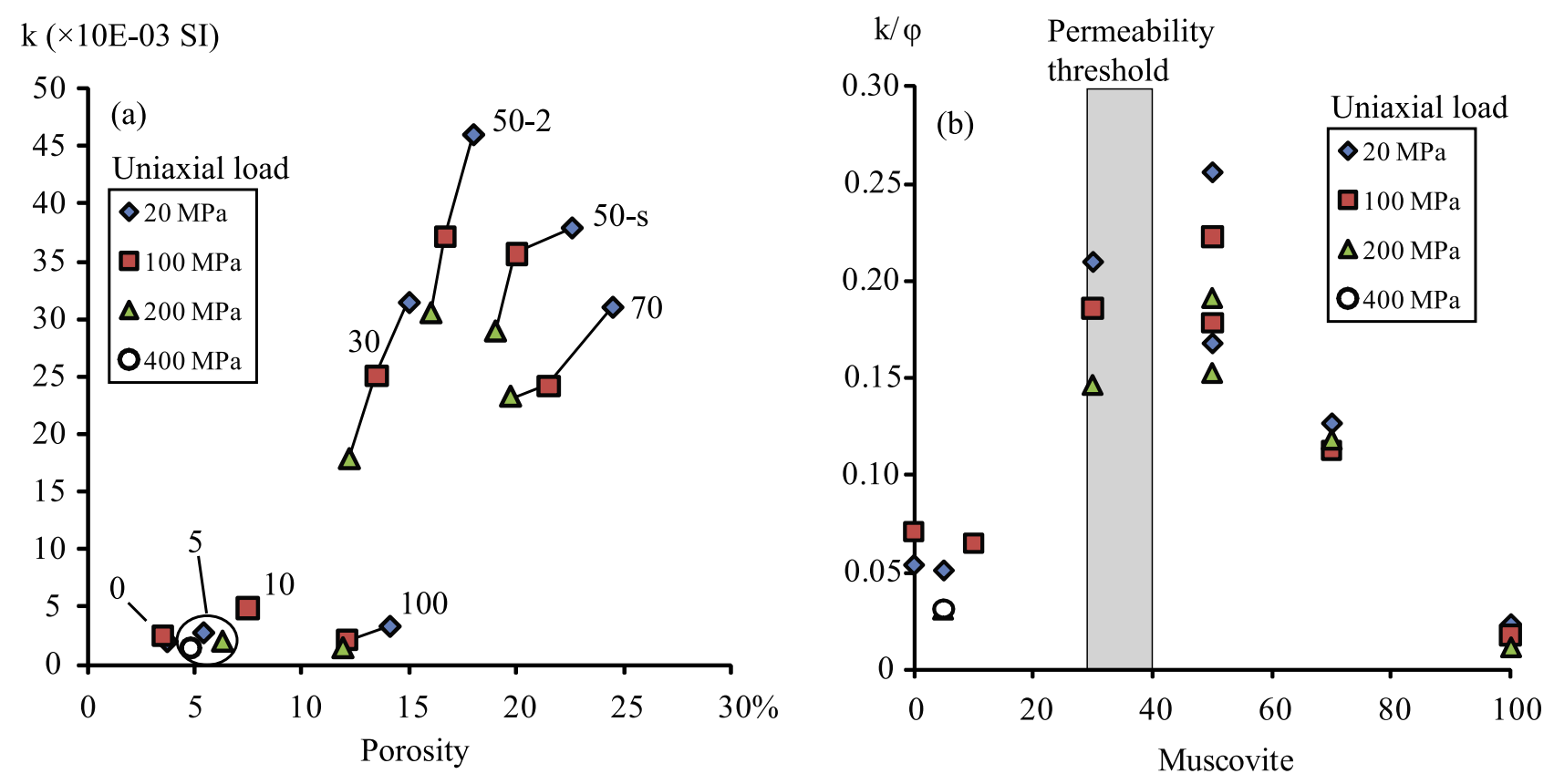

Figure 10. (a) Bulk susceptibility measured after ferrofluid impregnation as a function of the total sample porosity. Numbers next to the symbols indicate the percentage of muscovite for different uniaxial compaction loads used during manufacturing. (b) An illustration of the normalized bulk susceptibility with respect to porosity $(k / \varphi)$, as a function of muscovite percentage. The shaded inset box represents the 30-40\% kaolinite threshold observed by Crawford et al. [2008].

[33] The results presented here are supported by a study on the porosity and permeability of synthetic mixtures of quartz and kaolinite by Crawford et al. [2008], mixtures which served as analogs for fault gouges with varying clay contents. They observed a decrease in permeability which did not dominantly depend on the porosity amount, but instead on the ratio of kaolinite to quartz and effective pressure (i.e., the confining pressure minus the pore fluid pressure). At low kaolinite content, 20-40 vol \%, the effective pressure was the most important parameter for controlling the permeability, whereas for higher concentrations of kaolinite the impact of the clay particles themselves were more important for the observed permeability. In a plot of permeability as a function of clay content [cf. Crawford et al., 2008, Figure 9] they observe a threshold value for the change in slope of the permeability between 30 and $40 \mathrm{vol}$ $\%$ kaolinite, at which the dependence of permeability on clay content changes slope. Judging from the data in Figures 10a and $10 \mathrm{~b}$, it shows that samples containing between 30 and $50 \%$ muscovite display the highest magnetic susceptibility (i.e., permeability) with respect to the porosity amount, a threshold value that is in very good agreement with the results of Crawford et al. [2008]. Figure 10b shows the normalization of bulk susceptibility to porosity $(k / \varphi)$ as a function of vol \% muscovite mica, where the normalized value is indicative of permeability. The shaded box in Figure 10 outlines the permeability threshold of Crawford et al. [2008].

[34] Another factor that does play a role for ferrofluid impregnation is the wettability of the porous calcitemuscovite aggregates with respect to the oil-based ferrofluid [e.g., Abdallah et al., 2007]. The degree of wettability can be assessed by observing the contact angle between a droplet of the pore fluid and the solid. If the surface is hydrophilic (i.e., fluid is attracted to the surface of the solid), the droplet will spread across the solid surface producing a high contact angle, whereas if the surface is hydrophobic, the droplet will minimize its contact area to the surface and the contact angle will be small. The wettability affects the imbibition of the sample. Carbonate surfaces tend to be positively charged and therefore prone to be oil wet, having a large contact angle and therefore high wettability with respect to oil [Leslie Zhang et al., 2006]. In contrast, mica has negative surface charge and therefore displays a smaller contact angle with respect to the oil-based ferrofluid [Liu and Buckley, 1999]. Increasing amounts of mica will decrease the wettability of the solid surface, and in such a way potentially decrease the permeability of the synthetic samples.

\section{Conclusions}

[35] Effective medium calculations are used to predict elastic properties of materials. Many different theoretical treatments exist, including Voigt, Reuss and Hill estimates, Hashin-Shtrikman bounds, and self-consistent approximations. A type of self-consistent approximation has been applied in the current work, which considers the interaction of two materials, one being the background matrix and the second being the inclusion. Inclusions are incrementally added to the background matrix, whereby the effective material properties are continuously updated; the differential effective medium (DEM). It is possible to consider a background material with triclinic or higher order of symmetry, 
and inclusions of a specific ellipsoidal shape and orientation with respect to the background matrix. Although the DEM is not suitable for inclusions of fluids, since it is considered as a high-frequency model, it is possible to calculate the effective medium for a dry material, and subsequently substitute the fluid using the Biot-Gassmann fluid substitution equation [e.g., Le Ravalec and Guéguen, 1996; Mainprice, 1997].

[36] The DEM has been applied to predict the elastic properties of synthetic aggregates composed of mixtures of calcite and muscovite. The method of fabrication of the synthetic aggregates results in an anisotropic medium with varying porosity depending strongly on the ratio of calcite to muscovite. Using neutron diffraction texture goniometry the orientation distribution functions for calcite and muscovite were determined, and from these it is possible to calculate the composite elastic properties for the pore-free background matrix. Inclusions are added incrementally as empty dry pores. Pore shape is inferred from the AMS of samples impregnated with a ferrofluid. The ferrofluid is strongly magnetic, and its anisotropy of magnetic susceptibility provides the volumetric average aspect ratio of the pores and cracks that make up the porosity of the samples. Pore shapes vary as a consequence of the calcite to muscovite ratio, and uniaxial stress used to compact the synthetic samples.

[37] The predicted elastic properties for the synthetic samples are compared with laboratory acoustic measurements. Predicted velocities are consistently overestimated if considering only the elastic properties and symmetry of the pore-free background matrix. Predicted and measured seismic velocities agree well when inclusions with preferred shape are introduced using the DEM. Pore shape inferred from measurements of magnetic susceptibility of ferrofluid filled samples produce ellipsoids with aspect ratios ranging between 1.01:1 $(\mathrm{a}=\mathrm{b} \geq \mathrm{c})$ and 1.5:1. In order to match the measured $V_{P}$, an aspect ratio of the pores between $3: 1$ and 4:1 is needed. The aspect ratio determined from a model based on X-ray tomography for a specimen with $70 \%$ muscovite and compacted with $20 \mathrm{MPa}$ load is $\sim 2.2: 1$, falling between the ferrofluid and DEM predictions. Determining the pore shape using ferrofluid measurements is best utilized for samples with high porosity and permeability and together with the DEM provides a powerful combination to predict elastic properties in elastically anisotropic media.

[38] For rocks containing small volumes of cracks or pores, such as in the case of most crystalline rocks, high aspect ratios $(\alpha>10)$ have the strongest impact on the anisotropy of $P$ waves, although the impact is comparatively smaller with respect to maximum shear wave splitting (i.e., $V_{S}$ propagating parallel to the plane of the crack). In contrast spherical pores at low crack volumes have a weak influence on $V_{P}$ and $V_{S}$ anisotropy. At higher volumes of pores, relevant for reservoir rocks (20-40\%), smaller aspect ratios (between 1 and 5) produce significant $V_{P}$ anisotropy and $d V_{S}$. With pore volumes $>40 \%, d V_{S}$ appear to become particularly important, such as for loosely consolidated sediments, chalk and mudrocks.

[39] Acknowledgments. This work was supported by the Swiss National Science Foundation, project 2-77070-07. The authors wish to thank Claudio D'addario and Robert Hoffmann for technical help in the laboratory for natural magnetism and the rock deformation laboratory at ETH Zürich. This work benefited from critical reviews by Philip Benson and Dave Healy, as well as the Associate Editor Dan Faulkner. Sebastian Friess performed X-ray tomography measurements.

\section{References}

Abdallah, W., et al. (2007), Fundamentals of wettability, Oilfield Rev., 19, 44-61.

Almqvist, B. S. G., L. Burlini, D. Mainprice, and A. M. Hirt (2010), Elastic properties of anisotropic synthetic calcite-muscovite aggregates, J. Geophys. Res., 115, B08203, doi:10.1029/2009JB006523.

Anderson, D. L., B. Minster, and D. Cole (1974), The effect of oriented cracks on seismic velocities, J. Geophys. Res., 79, 4011-4015, doi:10.1029/JB079i026p04011.

Avseth, P., T. Mukerji, and G. Mavko (2005), Quantitative Seismic Interpretation, 359 pp., Cambridge Univ. Press, New York, doi:10.1017/ CBO9780511600074.

Barnett, D. M. (1972), The precise evaluation of derivatives of the anisotropic elastic Green's functions, Phys. Status Solidi C, 49, 741-748, doi:10.1002/pssb.2220490238.

Benson, P. M., P. G. Meredith, and E. S. Platzman (2003), Relating pore fabric geometry to acoustic and permeability anisotropy in Crab Orchard Sandstone: A laboratory study using ferrofluid, Geophys. Res. Lett., 30 (19), 1976, doi:10.1029/2003GL017929.

Benson, P., A. Schubnel, S. Vinciguerra, C. Trovato, P. Meredith, and R. P. Young (2006a), Modeling the permeability evolution of microcracked rocks from elastic wave velocity inversion at elevated isostatic pressure, J. Geophys. Res., 111, B04202, doi:10.1029/2005JB003710.

Benson, P. M., P. G. Meredith, and A. Schubnel (2006b), Role of void space geometry in permeability evolution in crustal rocks at elevated pressure, J. Geophys. Res., 111, B12203, doi:10.1029/2006JB004309.

Berryman, J. G. (2000), Seismic velocity decrement ratios for regions of partial melt in the lower mantle, Geophys. Res. Lett., 27, 421-424, doi:10.1029/1999GL008402.

Biot, M. A. (1956), Theory of propagation of elastic waves in a fluid saturated porous solid: 1. Low frequency range, J. Acoust. Soc. Am., 28, 168-178, doi:10.1121/1.1908239.

Bruner, W. M. (1976), Comment on "Seismic velocities in dry and saturated cracked solids" by Richard J. O'Connell and Bernard Budiansky, J. Geophys. Res., 81, 2573-2576, doi:10.1029/JB081i014p02573.

Chen, C.-C., C.-C. Lin, L.-G. Liu, S. V. Sinogeikin, and J. D. Bass (2001), Elasticity of single-crystal calcite and rhodochrosite by Brillouin spectroscopy, Am. Mineral., 86, 1525-1529.

Crawford, B. R., D. R. Faulkner, and E. H. Rutter (2008), Strength, porosity, and permeability development during hydrostatic and shear loading of synthetic quartz-clay fault gouge, J. Geophys. Res., 113, B03207, doi:10.1029/2006JB004634.

Dandekar, D. P. (1968), Pressure dependence of the elastic constants of calcite, Phys. Rev., 172, 873-877, doi:10.1103/PhysRev.172.873.

Eshelby, J. D. (1957), The determination of the elastic field of an ellipsoidal inclusion, and related problems, Proc. R. Soc. London, Ser. A, 241, 376-396, doi:10.1098/rspa.1957.0133.

Faccenda, M., L. Burlini, T. V. Gerya, and D. Mainprice (2008), Faultinduced seismic anisotropy by hydration in subducting oceanic plates, Nature, 455, 1097-1100, doi:10.1038/nature07376.

Gassmann, F. (1951), Über die elastizität poröser medien, Vierteljahrsschr. Naturforsch. Ges. Zuerich, 96, 1-23.

Healy, D., S. M. Ready, N. E. Timms, E. M. Gray, and A. V. Brovarone (2009), Trench-parallel fast axes of seismic anisotropy due to fluid-filled cracks in subducting slabs, Earth Planet. Sci. Lett., 283, 75-86, doi:10.1016/j.eps1.2009.03.037.

Henyey, F., and N. Pomphrey (1982), Self-consistent elastic moduli of a cracked solid, Geophys. Res. Lett., 9, 903-906, doi:10.1029/ GL009i008p00903.

Hill, R. (1952), The elastic behavior of a crystalline aggregate, Proc. Phys. Soc. London, Sect. A, 65, 349-354, doi:10.1088/0370-1298/65/5/307.

Hrouda, F., J. Hanák, and I. Terzijski (2000), The magnetic and pore fabrics of extruded and pressed ceramic models, Geophys. J. Int., 142, 941-947, doi:10.1046/j.1365-246x.2000.00216.x.

Jelinek, V. (1981), Characterization of the magnetic fabric of rocks, Tectonophysics, 79, T63-T67, doi:10.1016/0040-1951(81)90110-4.

Jezek, J., and F. Hrouda (2007), A program for magnetic susceptibilityequivalent pore conversion, Geochem. Geophys. Geosyst., 8, Q10013, doi:10.1029/2007GC001709.

Jones, S., P. Benson, and P. Meredith (2006), Pore fabric anisotropy: Testing the equivalent pore concept using magnetic measurements on synthetic voids of known geometry, Geophys. J. Int., 166, 485-492, doi:10.1111/j.1365-246X.2006.03021.x. 
Kendall, J.-M., and P. G. Silver (1996), Constraints from seismic anisotropy on the nature of the lowermost mantle, Nature, 381, 409-412, doi: $10.1038 / 381409 \mathrm{a} 0$.

Le Ravalec, M., and Y. Guéguen (1996), High and low-frequency elastic moduli for a saturated porous/cracked rock: Differential self-consistent and poroelastic theories, Geophysics, 61, 1080-1094, doi:10.1190/ 1.1444029.

Le Ravalec, M., Y. Guéguen, and T. Chelidze (1996), Magnitude of velocity anomalies prior to earthquakes, J. Geophys. Res., 101, 11,217-11,223, doi:10.1029/96JB00530.

Leslie Zhang, D., S. Liu, M. Puerto, C. A. Miller, and G. J. Hirasaki (2006), Wettability alteration and spontaneous imbibition in oil-we carbonate formations, J. Petrol. Sci. Eng., 52, 213-226, doi:10.1016/ j.petrol.2006.03.009.

Liu, L., and J. S. Buckley (1999), Alteration of wetting of mica surfaces, J. Petrol. Sci. Eng., 24, 75-83, doi:10.1016/S0920-4105(99)00050-9.

Louis, L., C. David, V. Metz, P. Robion, B. Menéndez, and C. Kissel (2005), Microstructural control on the anisotropy of elastic and transport properties in undeformed sandstones, Int. J. Rock Mech. Min. Sci., 42, 911-923, doi:10.1016/j.ijrmms.2005.05.004.

Mainprice, D. (1990), A FORTRAN program to calculate seismic anisotropy from the lattice preferred orientation of minerals, Comput. Geosci., 16, 385-393, doi:10.1016/0098-3004(90)90072-2.

Mainprice, D. (1997), Modeling the anisotropic seismic properties of partially molten rocks found at mid-ocean ridges, Tectonophysics, 279, 161-179, doi:10.1016/S0040-1951(97)00122-4.

Mainprice, D. (2007), Seismic anisotropy of the deep Earth from a mineral and rock physics perspective, in Treatise on Geophysics, vol. 2, edited by G. D. Price, pp. 437-491, Elsevier, Amsterdam.

Mainprice, D., and M. Humbert (1994), Methods of calculating petrophysical properties from lattice preferred orientation data, Surv. Geophys. 15, 575-592, doi:10.1007/BF00690175.

Mainprice, D., and A. Nicolas (1989), Development of shape and lattice preferred orientations: Application to the seismic anisotropy of the lower crust, J. Struct. Geol., 11, 175-189, doi:10.1016/0191-8141(89)90042-4.

Mallon, A. J., R. E. Swarbrick, and T. J. Katsube (2005), Permeability of fine-grained rocks: New insights from chalks, Geology, 33, 21-24, doi:10.1130/G20951.1.

Mavko, G., T. Mukerji, and J. Dvorkin (2009), The Rock Physics Handbook, 511 pp., Cambridge Univ. Press, New York, doi:10.1017/ CBO9780511626753.

McLaughlin, R. A. (1977), A study of the differential scheme for composite materials, Int. J. Eng. Sci., 15, 237-244, doi:10.1016/0020-7225(77) 90058-1.

Mura, T. (1987), Micromechanics of Defects in Solids, 614 pp., Martinus Nijhoff, Dordrecht.

O'Connell, R. J., and B. Budiansky (1974), Seismic velocities in dry and saturated cracked solids, J. Geophys. Res., 79, 5412-5426, doi:10.1029/JB079i035p05412.

Osborn, J. A. (1945), Demagnetizing factors of the general ellipsoid, Phys. Rev., 67, 351-357, doi:10.1103/PhysRev.67.351.

Pfleiderer, S., and H. Halls (1990), Magnetic susceptibility anisotropy of rocks saturated with ferrofluid: A new method to study pore fabric? Phys. Earth Planet. Inter., 65, 158-164, doi:10.1016/0031-9201(90) 90083-A.

Pfleiderer, S., and H. Halls (1993), Magnetic pore fabric analysis: Verification through image autocorrelation, J. Geophys. Res., 98, 4311-4316, doi:10.1029/92JB01851.
Pfleiderer, S., and H. Halls (1994), Magnetic pore fabric analysis: A rapid method for estimating permeability anisotropy, Geophys. J. Int., 116, 39-45, doi:10.1111/j.1365-246X.1994.tb02125.x.

Pfleiderer, S., and C. Kissel (1994), Variation of pore fabric across a foldthrust structure, Geophys. Res. Lett., 21, 2147-2150, doi:10.1029/ 94GL01907.

Reuss, A. (1929), Berechnung der fliessgrenze von mischkristallen aufgrund der plastizitätsbedingung für einkristalle, Z. Angew. Math. Mech., 9, 49-58, doi:10.1002/zamm.19290090104.

Røgen, B., I. Fabricius, P. Japsen, C. Høier, G. Mavko, and J. M. Pedersen (2001), Ultrasonic velocities of chalk samples: Influence of porosity, fluid content, and texture, paper presented at Stanford Rockphysics and Borehole Summer Meeting, Stanford Univ., Stanford, Calif., E1-1-E1-13.

Schmidt, V., L. Burlini, A. M. Hirt, and B. Leiss (2008), Fabrication of synthetic calcite-muscovite rocks with variable texture: An analogue to cataclasite fabrics, Tectonophysics, 449, 105-119, doi:10.1016/j. tecto.2007.11.055.

Schmidt, V., A. M. Hirt, B. Leiss, L. Burlini, and J. M. Walter (2009), Quantitative correlation of texture and magnetic anisotropy of compacted calcite muscovite aggregates, J. Struct. Geol., 31, 1062-1073, doi:10.1016/j. jsg.2008.11.012.

Stacey, F. D. (1960), Magnetic anisotropy of igneous rocks, J. Geophys. Res., 65, 2429-2442, doi:10.1029/JZ065i008p02429.

Stoner, E. C. (1945), The demagnetizing factors for ellipsoids, Philos. Mag., 36, 803-821.

Urmos, J., and R. H. Wilkens (1993), In situ velocities in pelagic carbonates: New insights from ocean drilling program leg 130, Ontong Java Plateau, J. Geophys. Res., 98, 7903-7920, doi:10.1029/93JB00013.

Uyeda, S., M. D. Fuller, J. C. Belshe, and R. W. Girdler (1963), Anisotropy of magnetic susceptibility of rocks and minerals, J. Geophys. Res., 68, 279-291, doi:10.1029/JZ068i001p00279.

Vaughan, M. T., and S. Guggenheim (1986), Elasticity of muscovite and its relationship to crystal structure, J. Geophys. Res., 91, 4657-4664, doi:10.1029/JB091iB05p04657.

Voigt, W. (1928), Lehrbuch der Kristallphysik, 978 pp.,Teubner, Leipzig, Germany.

Voltolini, M., H.-R. Wenk, N. H. Mondol, K. Bjørlykke, and J. Jahren (2009), Anisotropy of experimentally compressed kaolinite-illite-quartz mixtures, Geophysics, 74, D13, doi:10.1190/1.3002557.

Walsh, J. B. (1969), New analysis of attenuation in partially melted rock, J. Geophys. Res., 74, 4333-4337, doi:10.1029/JB074i017p04333.

Wenk, H. R., T. Takeshita, R. Jeanloz, and G. C. Johnson (1988), Development of texture and elastic anisotropy during deformation of hcp metals, Geophys. Res. Lett., 15, 76-79, doi:10.1029/GL015i001p00076.

Wenk, H.-R., M. Voltolini, H. Kern, T. Popp, and M. Mazurek (2008), Anisotropy in shale from Mont Terri, Lead. Edge (Tulsa Okla.), 27, 742-748, doi:10.1190/1.2944161

Willis, J. R. (1977), Bounds and self-consistent estimates for the overall properties of anisotropic composites, J. Mech. Phys. Solids, 25, 185-202, doi:10.1016/0022-5096(77)90022-9.

B. S. G. Almqvist and A. M. Hirt, Institute of Geophysics, ETH Zurich, Sonneggstrasse 5, CH-8092, Zurich, Switzerland. (bjarne.almqvist@erdw. ethz.ch)

L. Burlini and C. Madonna, Geological Institute, ETH Zurich, Sonneggstrasse 5, CH-8092, Zurich, Switzerland.

D. Mainprice, Géosciences Montpellier, Université Montpellier 2, Place Eugène Bataillon, FR-34095, Montpellier, France. 